Purdue University Purdue e-Pubs

2014

\title{
Manifold Microchannel Heat Sink Design Using Optimization Under Uncertainty
}

\author{
S Sarangi \\ Purdue University \\ K. K. Bodla \\ Purdue University \\ Suresh V. Garimella \\ Purdue University, sureshg@purdue.edu \\ J. Y. Murthy \\ University of Texas at Austin
}

Follow this and additional works at: http://docs.lib.purdue.edu/coolingpubs

Sarangi, S; Bodla, K. K.; Garimella, Suresh V.; and Murthy, J. Y., "Manifold Microchannel Heat Sink Design Using Optimization Under Uncertainty" (2014). CTRC Research Publications. Paper 202.

http://dx.doi.org/http://dx.doi.org/10.1016/j.ijheatmasstransfer.2013.09.067

This document has been made available through Purdue e-Pubs, a service of the Purdue University Libraries. Please contact epubs@purdue.edu for additional information. 


\title{
Manifold Microchannel Heat Sink Design Using Optimization Under Uncertainty
}

\author{
Suchismita Sarangi ${ }^{1}$, Karthik K. Bodla ${ }^{1}$, Suresh V. Garimella ${ }^{1, *}$ and Jayathi Y. Murthy ${ }^{2}$ \\ ${ }^{1}$ School of Mechanical Engineering and Birck Nanotechnology Center \\ Purdue University, 585 Purdue Mall, West Lafayette, IN 47907 USA \\ ${ }^{2}$ Department of Mechanical Engineering \\ The University of Texas at Austin, Austin, TX 78712 USA
}

\begin{abstract}
A three-dimensional numerical model is developed and validated to study the effect of geometric parameters such as microchannel depth and width, manifold depth, and manifold inlet and outlet lengths on the performance of a manifold microchannel (MMC) heat sink. The manifold arrangement used to distribute the flow through alternating inlet and outlet pairs greatly reduces the pressure drop incurred in conventional fluid supply arrangements due to its shorter flow paths, while simultaneously enhancing the heat transfer coefficient by limiting the growth of thermal boundary layers. The computational analysis is performed on a simple unit-cell model to obtain an optimized design for uniform thermal boundary conditions, as well as on a porous-medium model to obtain a complete system-level analysis of multiple microchannels across one manifold. The porous-medium approach can be further modified to analyze the performance under asymmetrical heating conditions. Along with conventional deterministic optimization, a probabilistic optimization study is performed to identify the optimal geometric design parameters that maximize heat transfer coefficient while minimizing pressure drop for an MMC heat sink. In the presence of uncertainties in the geometric and operating parameters of the system, this probabilistic optimization approach yields a design that is robust and reliable, in addition to being optimal. Such an optimization analysis provides a quantitative estimate of the allowable uncertainty in input parameters for acceptable uncertainties in the relevant output parameters. The approach also yields information such as the local and global sensitivities which are used to identify microchannel width and manifold inlet length as the critical input parameters to which the outputs are most sensitive. The deterministic analysis shows that the heat transfer performance of the MMC heat sink is optimal at a manifold inlet to outlet length ratio of 3. A comparison between the deterministic and probabilistic optimization approaches is presented for the unit-cell model. A probabilistic optimization study is performed for the porous-medium model and the results thus obtained are compared with those of the unit-cell model for a uniform heat flux distribution.
\end{abstract}

* Corresponding author: Tel. 7654945621 ; sureshg@purdue.edu 


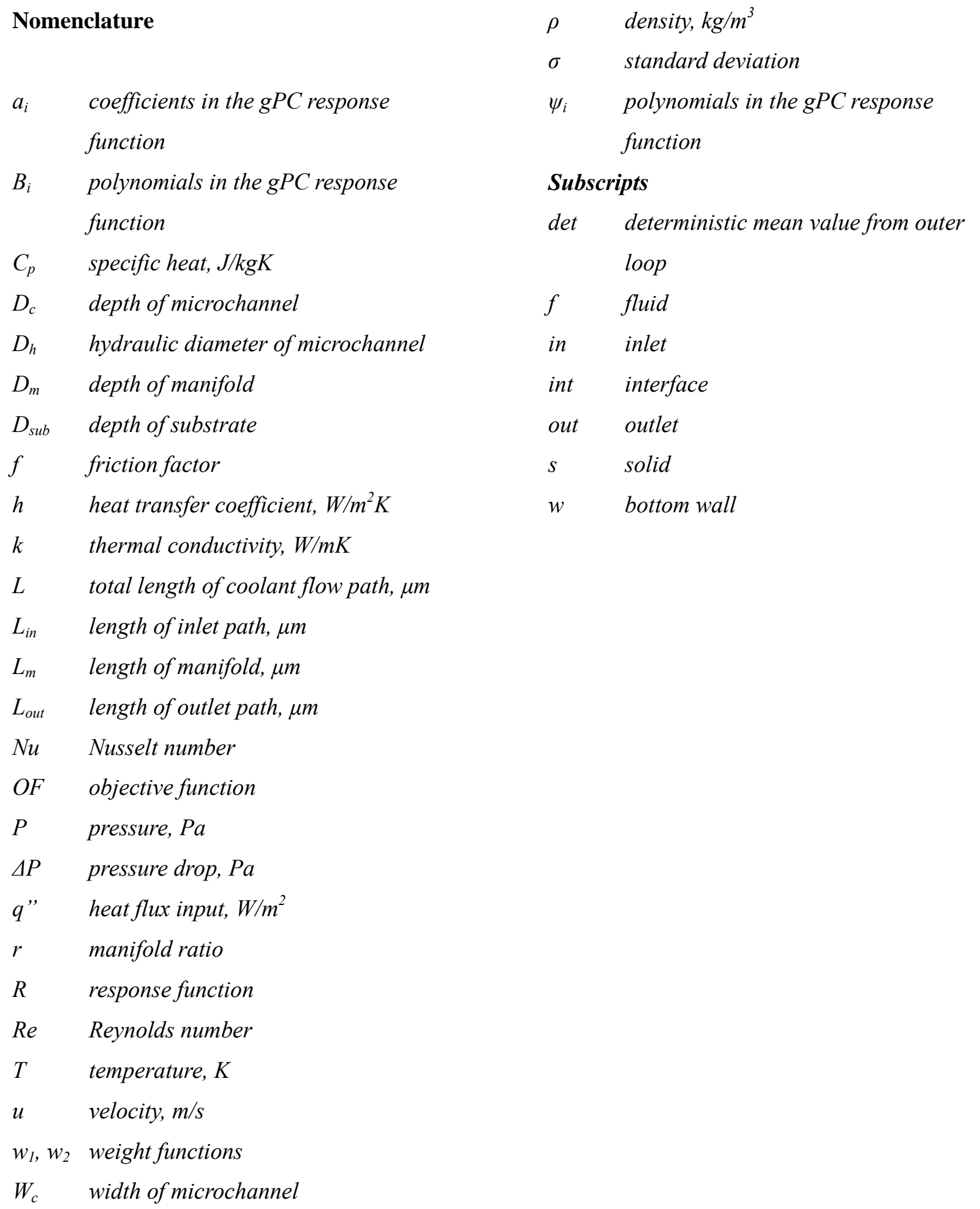

\section{Greek symbols}
$\alpha_{i} \quad$ coefficients in the response function
$\xi_{i} \quad$ random variable
$\mu \quad$ dynamic viscosity, $\mathrm{Ns} / \mathrm{m}^{2}$


Keywords: manifold, microchannel, uncertainty quantification, optimization under uncertainty, DAKOTA, generalized polynomial chaos, sensitivity analysis

\section{Introduction}

Conventional microchannel heat sinks are characterized by long microchannels that run parallel to the base of the heat sink, as proposed by Tuckerman and Pease [1]. These microchannel heat sinks have been successfully investigated for use in electronics cooling applications [2]. Several analytical and numerical models for predicting pressure drop and heat transfer through such heat sinks have been proposed in the literature [3-5]. Although conventional microchannels provide substantial heat transfer augmentation, they are also associated with very high pressure drops. Microchannel heat sinks with various modified configurations have been previously studied for improved performance over conventional single-layered rectangular microchannels. Deterministic optimization studies have been performed on microchannel heat sinks with double-layered channels [6] and tapered channels [7] to obtain optimum geometric parameters. An alternative configuration that has been proposed to lower the incurred pressure drop while simultaneously increasing the heat transfer is the manifold microchannel (MMC) heat sink. An MMC heat sink consists of a manifold system which distributes the coolant via multiple inlet-outlet pairs, thereby reducing the flow length of the coolant through the microchannels, as shown in Figure 1 (a). Such an arrangement results in a significant reduction in the pressure drop, while also reducing the thermal resistance by interrupting the growth of thermal boundary layers. This design was originally proposed by Harpole and Eninger [8], who demonstrated a significant reduction in thermal resistance relative to conventional microchannel heat sinks at a constant pumping power. Their MMC system consisted of 10 to 30 manifolds spanning the entire flow length. Kermani [9] and Kermani et al. [10] performed experiments to demonstrate the use of MMC heat sinks to cool concentrated solar cells, and reported a significant increase in heat transfer coefficient as compared to conventional microchannel heat sinks of similar dimensions. Experimental investigations were also reported by Copeland et al. [11] who observed the thermal resistance to be inversely proportional to the volume flow rate of the coolant. Kim et al. [12] demonstrated a 35\% reduction in thermal resistance relative to a conventional arrangement for forced air cooling. Copeland et al. [13] conducted a simplified 1D analysis to predict the pressure drop and thermal resistance of MMC heat sinks based on correlations for a straight rectangular channel. This analytical model was reasonably accurate at high flow rates, but was found to be inadequate for the geometry under consideration at low flow rates. The thermal resistance obtained using the analytical model was found to be about $50-70 \%$ lower than that predicted using a simplified 3-D isothermal numerical model. Ryu et al. [14] performed a detailed 3-D numerical analysis for quantifying the thermal 
performance of an MMC heat sink configuration, and included a consideration of the manifold depth and the bottom solid wall which were previously excluded from analysis [13]. Further, an optimization study was also performed using the steepest-descent method for arriving at the optimal design that would yield the minimum thermal resistance at different pumping powers. It was observed that the optimal geometric parameters as well as the optimal thermal resistance had a power-law dependence on the pumping power.

Microchannel-based heat sinks involve uncertainties in a number of parameters, such as those due to inherent limitations of the fabrication technique, and in the operating conditions such as the inlet flow rate and the input heat fluxes. In the presence of such uncertainties, a conventional, deterministic optimization approach may not provide a truly optimized design that is also robust. The challenge is to quantify these uncertainties and include them in the optimization procedures to produce designs meeting the desired reliability and robustness levels. The typical approach in the presence of such uncertainties is to perform an optimization by considering the uncertain variables as design parameters fixed at their mean values (or at bounds), and then include a post-design factor of safety to produce a conservative design that accounts for uncertainties heuristically. However, in the presence of large uncertainties or when there are strict constraints (such as expensive design parts, designs with constraints on overall mass, etc.), this approach fails to produce a truly optimal design. Instead, it merely provides a heuristic, conservative estimate and does not involve explicit quantification of the output uncertainties relative to input uncertainties.

An improved alternative strategy is to adopt a probabilistic optimization approach using the optimization under uncertainty (OUU) method which combines the optimization procedure with uncertainty quantification (UQ) [15] . Optimization under uncertainty is a powerful approach that ensures reliable design of systems by considering the input uncertainties as part of the design process. The OUU approach may be used for "design for reliability" and for "design for robustness" [16]. The goal of the former problem is to produce an optimized design with a reduced probability of failure, while in the latter case, the objective is to produce designs that are less sensitive to variable inputs. As discussed by Eldred et al. [16], the latter problem is often the simpler one to address and does not always require UQ. However, non-UQ-based approaches rely on local derivatives to assess robustness and are hence not recommended. In the design for reliability problem, UQ is performed and design parameters are estimated based on the tail (end) statistics of the output probability density function (PDF). This problem places a greater demand on the UQ and is often computationally more expensive compared to the design for robustness problem.

The objective of the present study is to optimize the geometry of a manifold microchannel heat sink based on a probabilistic approach to account for the inherent uncertainties in fabrication and operating conditions. A 3-D numerical unit-cell model for the MMC heat sink is first developed and validated by comparing against previous experimental results [9]. The geometric parameters and input conditions for 
the model are similar to those used in the experiments. Further, a 3-D porous-medium model is also developed to perform a system level analysis and optimization. In this model, the microchannel heat sink is represented as a porous medium with equivalent porosity and permeability [17], so as to reduce computational complexity. After validating the numerical model, an OUU analysis is performed by using a nested approach as discussed in detail in [15]. In this approach, uncertainty quantification is performed in an inner loop, which is nested within an outer optimization loop, as described in section 3.2.

The cost incurred by multiple CFD simulations is alleviated by using a generalized polynomial chaos (gPC) based response surface approach for the inner loop UQ [18]. Robust optimal designs, restricting the standard deviation of the output - the heat transfer coefficient - are obtained for a variety of cases. Further, the computed results are compared against those obtained via a traditional deterministic approach, i.e., an optimization study with mean values assumed for the uncertain variables. The OUU analysis is also performed for the porous-medium model using the same geometric parameters and optimization conditions and constraints, and the results are compared against those obtained from the probabilistic optimization using the unit-cell model.

\section{Numerical Modeling}

This section describes the numerical modeling approach employed in the current work. The models are described only briefly here, and a detailed discussion on the UQ and OUU methodology employed may be found in Bodla et al. [15]. In the present study, two sets of computations are performed. In the first, a unit-cell domain is employed for a detailed numerical analysis of the fluid-flow and heat transfer, and the results are compared with past experiments; the performance of the unit cell is optimized using traditional deterministic as well as the probabilistic (OUU) approaches discussed above. Following this, a complete system-level analysis is also performed using a porous-medium model, wherein the simulations are performed over several microchannels throughout the length of one manifold. A porous-medium treatment of the microchannel heat sink is employed in this analysis using a two-temperature non-thermal equilibrium model. The two kinds of computations are discussed below.

\subsection{Unit-Cell Model}

A schematic diagram of the MMC heat sink considered in the present work is shown in Figure 1 (a). The manifold distribution system is placed on top of the microchannels, in a direction transverse to the main flow direction. The coolant is pumped in through a common inlet port, which branches out into parallel manifold inlet channels. Upon entering the microchannel, the fluid undergoes a 90-degree turn, traverses along the microchannel length removing heat from the substrate, and subsequently flows through another 90-degree turn and then exits upward through the outlet manifold channels and into the 
common outlet port. Exploiting symmetry and periodicity, only a unit cell of the MCC heat sink is considered for detailed numerical analysis. Figure 1(b) shows the unit cell employed, along with the applicable boundary conditions. The continuity, momentum, and energy equations for steady-state, laminar, incompressible flow with constant thermophysical properties may be written as [19]:

$$
\begin{gathered}
\frac{\partial}{\partial x_{j}}\left(\rho u_{j}\right)=0 \\
\frac{\partial}{\partial x_{j}}\left(\rho u_{j} u_{i}\right)=-\frac{\partial P}{\partial x_{i}}+\frac{\partial}{\partial x_{j}}\left(\mu_{f} \frac{\partial u_{i}}{\partial x_{j}}\right) \\
\frac{\partial}{\partial x_{j}}\left(\rho u_{j} C_{p} T_{f}\right)=\frac{\partial}{\partial x_{j}}\left(k_{f} \frac{\partial T_{f}}{\partial x_{j}}\right) \quad(\text { liquid }) \\
0=\frac{\partial}{\partial x_{j}}\left(k_{s} \frac{\partial T_{s}}{\partial x_{j}}\right) \quad(\text { solid) }
\end{gathered}
$$

Here $u_{j}, P$, and $T$ represent the velocity, pressure and temperature fields, while the subscripts $s$ and $f$ refer to the solid and fluid medium, respectively. Without loss of generality, water is used as the coolant in the current study and the walls of the microchannel and manifold are assumed to be made of silicon. The low Reynolds numbers considered for the flow justify the assumption of laminar, steady flow.

At the inlet, a constant mass flow condition is specified with the fluid entering at a constant temperature of $T_{i n}=300 \mathrm{~K}$, while a constant pressure condition is imposed at the outlet. Symmetry conditions are specified for both velocity and temperature at the four outer boundary planes, i.e., at $x=0$, $x=x_{\max }$, and at $z=0$, and $\mathrm{z}=z_{\max }$. A uniform heat flux of $q_{w}{ }^{\prime \prime}=100 \mathrm{~W} / \mathrm{cm}^{2}$ [14] is specified on the bottom wall of the substrate, while the top wall of the manifold is assumed to be adiabatic. Also, as the problem involves conjugate heat transfer, continuity of temperature and heat flux is employed at all the solid-fluid interfaces, as given by [20].

$$
\begin{aligned}
T_{s, \text { int }} & =T_{f, \text { int }} \\
-k_{s}\left(\frac{\partial T_{s}}{\partial n}\right)_{\text {int }} & =-k_{f}\left(\frac{\partial T_{f}}{\partial n}\right)_{\text {int }}
\end{aligned}
$$

The geometry is modeled and meshed using the mesh generation package CUBIT [21]. The governing equations along with the boundary conditions described are solved using the commercial CFD package FLUENT [22]. Pressure-velocity coupling is addressed via the SIMPLE algorithm, along with an algebraic multigrid algorithm (AMG) for solving the linearized system of governing equations. The governing equations are solved using a first-order upwind scheme to obtain an approximate solution that is employed as the initial condition for a more accurate, second-order upwind scheme analysis. The 
number of iterations for this initial first-order solution were chosen by observing the overall time reduction achieved relative to a purely second-order scheme without a precursor first-order solution. Allowing the scaled residuals with the initial first-order scheme to converge close to the final convergence criteria employed reduced the number of second-order iterations required. As a comparison, the overall solution time with this approach was observed to be approximately $60 \%$ of that required with just the second-order scheme employed from the beginning. Employing the flow and temperature fields so obtained as the initial conditions, the equations are then solved using a second-order upwind scheme until convergence, to obtain more accurate converged solutions. The use of the initial conditions obtained from the first-order scheme reduces the computational time required for second order convergence. The equations are also suitably under-relaxed, and convergence criteria of $10^{-6}$ for scaled residuals of continuity and momentum equations, and $10^{-9}$ for energy equation are specified. In addition, the average pressure at the inlet and the average temperature of the bottom wall are also monitored to check for convergence of the flow and energy equations.

\subsection{Porous-Medium Model}

Unit-cell models, though accurate, have certain limitations. First, owing to the underlying assumptions of periodicity, non-uniform heat fluxes such as those encountered in a realistic scenario cannot be incorporated. Second, modeling the entire heat sink using such an approach is not computationally feasible. In lieu of these limitations, a porous-medium model was developed for a system level flow and heat transfer analysis and optimization of the manifold microchannel heat sink.

Microchannel heat sinks have previously been modeled as equivalent porous medium successfully [17]. In this approach, the hydrodynamic performance of the heat sink is modeled via an equivalent permeability and porosity, without the need to resolve the heat sink geometry down to scale of individual fins and channels. For the current configuration, shown in Figure 1 (c), we employ the following parameters described in Liu and Garimella [17]:

$$
\begin{aligned}
& \varepsilon=\frac{W_{c}}{W_{c}+W_{f i n}} \\
& \kappa_{y, z}=\frac{\varepsilon W_{c}^{2}}{12} ; \kappa_{x}=\frac{\kappa_{y, z}}{100}
\end{aligned}
$$

In these definitions, $\varepsilon$ denotes the porosity of the microchannel and $W_{f i n}$ is the width of the fin, which in this study is assumed equal to the width of the microchannel, $W_{c}$. The permeability is anisotropic and is denoted by $\kappa$. The permeability in the x-direction, transverse to the main flow direction, is assumed to be lower by two orders of magnitude relative to the value in the flow direction (y and $\mathrm{z}$ ) so as to account for the impermeable walls of the microchannel. Further, the complexity of the problem is reduced by 
excluding the bottom wall of the heat sink (included in the unit-cell model) from the computational domain for the porous-medium model. The implications of this change are described in section 4.1.2. The hydrodynamic performance of such a porous medium may be modeled using Navier-Stokes equations in conjunction with Darcy's equation, as discussed in Escher et al. [20]:

$$
\frac{\partial}{\partial x_{j}}\left(\rho_{f} u_{j} u_{i}\right)=-\frac{\partial P}{\partial x_{i}}+\frac{\partial}{\partial x_{j}}\left(\mu_{f} \frac{\partial u_{i}}{\partial x_{j}}\right)-\frac{\mu_{f}}{\kappa_{i}} u_{i}
$$

Here, $\mu_{f}$ is the viscosity of the fluid. The last term on the right hand side of Eq. (8) denotes the additional viscous pressure drop (Darcy pressure drop), owing to the presence of the solid microchannel walls.

The thermal performance of the porous medium can be modeled using the two-temperature model as discussed by Kaviany [23] and Kim and Kim [24]. In this work, we employ this two-temperature model to account for the local thermal non-equilibrium between the solid and fluid phases within the control volume of the porous medium. The energy equations for the solid and fluid phases of the porous medium are thus modeled as follows:

$$
\begin{gathered}
k_{s e} \frac{\partial^{2}\langle T\rangle_{s}}{\partial y^{2}}=h_{s f} a_{s f}\left(\langle T\rangle_{s}-\langle T\rangle_{f}\right) \\
\varepsilon \rho_{f} C_{p, f} u_{f} \frac{\partial\langle T\rangle_{f}}{\partial x}=h_{s f} a_{s f}\left(\langle T\rangle_{s}-\langle T\rangle_{f}\right)+k_{f e} \frac{\partial^{2}\langle T\rangle_{f}}{\partial y^{2}} \\
k_{s e}=(1-\varepsilon) k_{s} ; k_{f e}=\varepsilon k_{f}
\end{gathered}
$$

Here, $a_{s f}$ is the wetted surface area per unit volume, while $k_{s e}$ and $k_{f e}$ are the effective thermal conductivities of the solid and fluid phases, respectively [17]. The volume-averaged temperatures of the solid and fluid phases are represented by $\langle T\rangle_{s}$ and $\langle T\rangle_{f}$, respectively. The right hand side of Eq. (9) represents the interfacial heat transfer and may be modeled via a suitable value for the interfacial heat transfer coefficient, $h_{s f}$. In this study, we employ a developing flow correlation for single-phase heat transfer in microchannels proposed by Lee and Garimella [5]. The interfacial heat transfer coefficient and the Nusselt number are dependent on location in the flow direction, and are given as follows:

$$
\begin{aligned}
& h_{s f, z}=\frac{N u_{z} k_{f}}{D_{h}} \\
& N u_{z}=\frac{1}{C_{1}\left(z^{*}\right)^{C_{2}}+C_{3}}+C_{4}
\end{aligned}
$$

In this equation, $z^{*}$ denotes the non-dimensional axial distance from the inlet, and $C_{1}, C_{2}, C_{3}$ and $C_{4}$ are empirical constants. The values of the empirical constants, and details on limitations and applicability of the correlation may be found in Lee and Garimella [5]. 
The computational domain shown in Figure 1 (c) is meshed using the mesh generation package CUBIT [21]. Again, exploiting symmetry, the computational domain consists of one half each of an inlet and outlet manifold in the flow direction, while the entire heat sink length is considered in the transverse direction. The use of such a porous-medium model allows a system-level analysis with reduced geometrical complexity, thereby providing greater flexibility at significantly reduced computational cost, as will be discussed in section 4.1.2. A constant mass flux boundary condition is applied at the inlet, while a constant pressure condition is imposed at the outlet. Further, a constant heat flux boundary condition is applied on the bottom wall of the microchannel section, simulating the heat source. All other interfaces are assumed to have a no-slip boundary condition along with temperature and heat flux continuity between the solid and fluid zones of the porous medium, as appropriate. The governing equations, along with the boundary conditions as described above, are solved to convergence, using the built-in porous-medium non-equilibrium thermal model in the commercial CFD package FLUENT [22]. Pressure-velocity coupling is addressed via the SIMPLE algorithm, along with an algebraic multigrid algorithm (AMG) for solving the linearized system of governing equations. For monitoring convergence, we employ a solution procedure similar to that employed for the unit-cell model, whereby the governing equations are first solved using a first-order upwind scheme for a few iterations. Employing the flow and temperature fields so obtained as the initial conditions, the equations are then solved using a second-order upwind scheme until convergence is achieved. The governing equations are also suitably under-relaxed to ensure proper convergence. Moreover, the average pressure at the inlet and the average temperature of the bottom wall are also monitored to check for convergence of the flow and energy equations, respectively.

\section{Solution Methodology}

The optimization methodology and the uncertainty quantification (UQ) procedure are described briefly. Further details may be found in Eldred [25] and Xiu and Karniadakis [18].

\subsection{Uncertainty Quantification}

The first step in the optimization under uncertainty procedure is uncertainty quantification (UQ). This procedure entails determination of uncertainties in outputs for given input uncertainties. Uncertainties are commonly categorized as being aleatoric or epistemic. The aleatoric uncertainties (also known as statistical uncertainties) in inputs result from an inherent randomness which occurs every time an experiment is run, while the epistemic uncertainties (also known as systematic uncertainties) result from limited data and knowledge [25]. In the present work, the analysis is restricted to aleatorically 
uncertain variables, for which probabilistic methods such as polynomial chaos expansions (PCEs) may be used to determine the output statistics.

The most common UQ methods used are random sampling techniques. Random sampling methods employ standard algorithms such as Monte Carlo or Latin Hypercube sampling, for randomly drawing samples based on input probability distribution functions. In this method, the simulation is performed for each sample drawn, and when the entire range of input variations is covered, response statistics and PDFs of outputs are computed $[16,23]$. This entails performing thousands of simulations to cover the entire the range of input variations. For complicated problems, this becomes untenable owing to the large number of simulations involved. Other methods such as the sensitivity method based on moments of samples are also used for UQ, but these methods are less robust and depend on the model assumptions.

For moderate numbers of input random variables, the polynomial chaos expansion (PCE) method is more efficient and computationally tractable than random sampling methods. In the present work, the generalized polynomial chaos (gPC) approach is used with the Wiener-Askey scheme [18] . In this approach, uncertain variables, represented by normal, uniform, exponential, beta, and gamma PDFs, are modeled by Hermite, Legendre, Laguerre, Jacobian and generalized Laguerre orthogonal polynomials, respectively. It has been shown that these orthogonal polynomials are optimal for the corresponding distribution types since the inner product weighting function and its support range correspond to the PDFs of these distributions [18]. In theory, this selection of the optimal basis allows for exponential convergence rates. The gPC method may be either intrusive or non-intrusive. The stochastic collocation method is a non-intrusive method based on gPC [18]. In this method, the polynomials mentioned above are used as an orthogonal basis to estimate the dependence of the stochastic form of the output on each of the uncertain inputs. Deterministic simulations are performed at the collocation points in random space. The coefficients in the polynomial expansion are determined by making use of the orthogonality properties of the polynomial basis function. Further details may be found in [18] and in the comprehensive review by Eldred [25]. The utility of such a non-intrusive gPC approach in the design of electronics cooling equipment such as pin-fin heat sinks, and its advantages compared to an intrusive approach, were demonstrated recently by Bodla et al. [15].

The polynomial chaos expansion for a response $R$ is expressed as

$$
R=\sum_{j=0}^{\infty} \alpha_{j} \psi_{j}(\xi)
$$

Each of the terms $\psi_{j}(\xi)$ consists of multivariate polynomials obtained from the products of the corresponding one-dimensional polynomials. Neglecting the higher-order terms in Eq. (13) results in a finite number of evaluations needed to compute the response function $R$. The Smolyak sparse grid technique can be used to select the specific evaluation points. This sparse grid technique has proven to be 
computationally more efficient than other methods such as quadrature grids for each random variable (which gives a tensor product grid when there are more than one random variables) [25]. The Smolyak sparse grid requires fewer computations than the tensor product grid when there are a large number of uncertain parameters. Hence, the Smolyak grid has been used for the present work.

Deterministic simulations are performed at the points selected by this method, and the response surface of the outputs is generated. This response surface is then used as a surrogate model for the dependence of the output on inputs. The PDFs of the response $R$ may be computed by sampling the space of input random variables using random sampling algorithms such as Monte Carlo or Latin Hypercube sampling. Output response statistics, such as PDFs, and the mean and standard deviation of the outputs, may then be readily computed [15].

The gPC-based UQ analysis also provides other useful information such as Sobol's indices [26]. Sobol's indices indicate the sensitivity of output parameters to the various uncertain input parameters; such information is valuable in identifying critical input parameters. The sensitivity information obtained from the Sobol indices from a coarse UQ analysis may be used to exclude some of the parameters which do not affect the outputs significantly. The subsequent refined UQ analysis can then be performed with fewer uncertain variables, thereby reducing the computational effort significantly. In the present study, the open source UQ and optimization toolkit, DAKOTA (Design Analysis Kit for Optimization and Terascale Applications) [27], is used for performing the UQ analysis as well as the corresponding optimization.

\subsection{Optimization Under Uncertainty}

Optimization under uncertainty (OUU) refers to probabilistic optimization, which involves optimization of a design by taking into consideration the uncertainties in inputs and the corresponding output response statistics. The optimization toolkit DAKOTA used in the present study consists of various OUU formulations, as described in detail by Eldred et al. [16]. In this work, the nested approach is used for the probabilistic optimization in which the UQ performed in the inner loop is nested within an outer optimization loop [18]. DAKOTA consists of various gradient and non-gradient based optimization algorithms; we choose the gradient-based Fletcher-Reeves conjugate gradient method for unconstrained optimization, and the method of feasible directions for constrained optimization. These tools are available in the CONMIN library [28] of the DAKOTA package.

The nested OUU approach employed in the present work is shown schematically in Figure 2. The initial guesses for the various design variables are provided by the user. Starting with these values, the gPC-based method described earlier is used to perform the complete UQ analysis in the inner loop for the specified uncertainties in the input parameters. The uncertain design variables generated from the outer 
optimization loop are mapped into the inner UQ loop as required, using nested model controls available in DAKOTA [14]. The output response metrics from the UQ loop are used to evaluate the statistics of the objective function such as the mean and standard deviation. These output statistics are then passed on to the optimizer in the outer loop. The optimizer verifies if the objective function is maximized (minimized), in addition to satisfying the various constraints that are imposed, such as those to restrict the standard deviation in an output. If the convergence criteria are not met, i.e., if a constraint is violated, or if the objective function is not at its maximum (or minimum), a new set of design variables is selected and the whole procedure is repeated. Thus, at convergence, the set of design variables that optimizes the objective function and simultaneously satisfies the specified constraints is obtained [15].

For performing probabilistic optimization effectively, the entire process involved in the nested loops as described above must be automated. This is achieved by using DPREPRO, the built-in pre-processor available in DAKOTA [27]. A simple Python script is written to automate the entire process shown in Figure 2. The geometry is parameterized for meshing, and the journal features of the meshing package CUBIT [21] are utilized for generating meshes at the Smolyak collocation points. Once the computational model is parameterized, actual values of the parameters for individual evaluations are obtained using DPREPRO with little or no manual intervention. The governing equations are solved using the commercial CFD package FLUENT [22]. To increase computational efficiency, the parallel CFD capabilities of FLUENT are employed. The various inner loop UQ evaluations at the Smolyak collocation points are also performed in parallel to reduce the overall computational time. The outputs from the FLUENT evaluations are generated in the format required by DAKOTA by the use of suitable user-defined functions. After the first outer-loop iteration, the results are passed back to the optimizer, which then decides the next set of design variables. The process is repeated until the convergence criterion and the constraints are satisfied. The OUU process described here has been validated for a simple heater block design and used for pin-fin heat sink optimization by Bodla et al. [15].

With the available computational resources and with the use of parallelized CFD solvers, each simulation (one complete inner loop evaluation) required approximately 90 minutes of real time for the unit-cell model and about 45 minutes of real time for the porous-medium model. The simulations were performed using 4 Intel E5410 processors in parallel. The Smolyak grid determines the number of inner loop simulations required for each outer loop set of design variables. A sparse grid of level 1 was used for the inner uncertainty loop for the probabilistic optimization, which resulted in 7 inner loop evaluations for 3 uncertain variables. The computational time can be reduced by first performing a deterministic optimization and then using the optimized values obtained as initial guess values for the probabilistic optimization. The optimization under uncertainty is first carried out for the unit-cell model and then repeated for the porous-medium model. 


\section{Results and Discussion}

We now present results for OUU of manifold microchannel heat sinks obtained via the unit-cell and the porous-medium models, respectively. The optimal designs obtained through deterministic and probabilistic optimizations, performed using the unit-cell model, are compared. Further, results of probabilistic optimization, obtained using the porous-medium model, are discussed.

\subsection{Verification and Validation}

Before performing the optimization, the numerical models are first verified by comparing against experimental results performed in the literature on geometrically-similar heat sinks. A meshindependence study is also performed prior to parameterizing the model for use in the automated OUU study.

\subsubsection{Unit-Cell Model}

For assessing mesh independence of the unit-cell model, the average pressure difference between the inlet and outlet ports is computed and compared for different grid sizes for a test case. The meshing is performed in CUBIT [22] employing the tetmesh scheme. Also, for lowering the overall mesh count, a graded mesh with a gradually increasing mesh size ratio, finer at the solid-fluid interface and coarser towards the bulk volume, is employed. The mesh size ratio is defined as the ratio of the cell size furthest from the heated boundary wall to that of the cell nearest to the boundary wall. By this means, the level of mesh refinement for which the percentage error with respect to the finest grid size falls below an acceptable value is selected as the optimum mesh size for all the subsequent evaluations. The results of the mesh independence study for the unit-cell model are shown in Figure 3 (a), performed for an MMC heat sink with parameters, $W_{c}=80 \mu \mathrm{m}, D_{c}=200 \mu \mathrm{m}, D_{m}=300 \mu \mathrm{m}, L_{\text {out }}=120 \mu \mathrm{m}, r=0.5$, at a fixed inlet mass flow rate of $0.5 \mathrm{~g} / \mathrm{s}$. For this case, a mesh size of 655,360 (40 x 64 x 256) cells, corresponding to a mesh size ratio of 6.25 , is observed to result in a pressure drop value which is within $0.3 \%$ of that obtained employing the finest grid size, consisting of about triple the number of mesh elements. Hence, results obtained via this mesh size may be deemed mesh-independent and this mesh is used for the results presented in this work.

To characterize flow and heat transfer phenomena and to validate the unit-cell model, simulations are first performed for fixed geometric parameters. Fluid is pumped through the inlet manifold of an MMC heat sink with fixed dimensions at varying mass flow rates, and a heat flux of $75 \mathrm{~W} / \mathrm{cm}^{2}$ is applied to the bottom surface. Figure 4 (a) shows the velocity vectors obtained at the center plane of microchannel. As the fluid enters the microchannel, due to the sudden contraction, it accelerates rapidly. The fluid turns 
through 90 degrees and travels through the channel. At the end of the manifold, the fluid again turns through 90 degrees and exits via the outlet. Figure 4 (b) and Figure 4 (c) show the thermal contours at the center plane for two different inlet mass flow rates of $0.5 \mathrm{~g} / \mathrm{s}$ and $5.0 \mathrm{~g} / \mathrm{s}$. It is observed that the maximum cooling effect is seen at the channel inlet region, where the thermal boundary layer is thinnest. Figure 4 (c) also shows the enhanced heat transfer obtained at higher flow rates.

In order to validate the numerical procedure, the heat transfer coefficient for various flow rates is compared with experimental results from Kermani [9]. The heat transfer coefficient is calculated as [9]:

$$
h=\frac{q_{w}^{\prime \prime}}{T_{w}-0.5\left(T_{\text {in }}+T_{\text {out }}\right)}
$$

Figure 5 shows the heat transfer coefficient values as a function of the flow rate, for the case of a channel with an aspect ratio $\left(W_{c} / D_{c}\right)$ of 0.1 . It may be observed that as the flow rate of the coolant increases, the heat transfer coefficient increases as expected. Further, the results from the present computations are found to be in close agreement with the experimental results of Kermani [9], within limits of the experimental uncertainties reported. This validates our numerical unit-cell model.

Having verified and validated the numerical model, simulations are performed to observe the effects of varying geometric parameters. For all subsequent simulations, fluid is pumped through the inlet manifold at an overall mass flow rate of $0.5 \mathrm{~g} / \mathrm{s}$ [9], and a heat flux of $100 \mathrm{~W} / \mathrm{cm}^{2}$ [14] is applied on the bottom wall, unless otherwise mentioned.

\subsubsection{Porous-Medium Model}

A mesh-independence study, similar to that for the unit-cell model, is also performed for the porousmedium model. For the inlet and outlet fluid volumes, a graded mesh, made finer near the solid-fluid and porous-fluid boundary interface walls and coarser away from these boundaries, is used, similar to the unit-cell model. The comparatively simple microchannel porous medium volume is meshed with coarse grids. A manifold length of $1000 \mu \mathrm{m}$ (equal to the heat sink size in the transverse direction) and a coolant flow rate of $0.5 \mathrm{~g} / \mathrm{s}$ is considered. Figure 3 (b) shows the results obtained from the mesh-independence study. The computed inlet-to-outlet pressure drop with a mesh size of 309,000 cells, corresponding to a mesh size ratio of 6.1 , was found to be within $0.3 \%$ of that obtained employing the finest mesh size, consisting of approximately 1,600,000 cells. For all the subsequent simulations, a mesh size of approximately 309,000 cells is used. For the unit-cell model, this manifold length of $1000 \mu \mathrm{m}$ corresponds to 25 microchannels of width $W_{c}=20 \mu \mathrm{m}$ each, with a mesh size of 655,360 cells per microchannel unit cell. The porous-medium model not only reduces the required mesh size by half, but also represents the full array of microchannels, unlike the single microchannels considered in the unit-cell model. 
Numerical computations with the porous-medium model are performed for same values of coolant flow rates and heat fluxes as in the case of the unit-cell model, to facilitate a one-to-one comparison of the models. Table 1 shows the pressure drop obtained with the unit-cell and porous-medium models, for different inlet flow rates and a constant channel width of $20 \mu \mathrm{m}$. Similarly, Table 2 (a) shows a comparison of pressure drops obtained by these models, computed for a variety of microchannel widths at a constant inlet mass flow rate of $0.5 \mathrm{~g} / \mathrm{s}$. As may be noted from Table 1 and Table 2 (a), the results obtained via the porous-medium model are within $8 \%$ of those obtained via the detailed unit-cell model. Nusselt number values at an imposed heat flux of $100 \mathrm{~W} / \mathrm{cm}^{2}$ are also computed and compared. Table 2 (b) shows the variation of the average Nusselt number $N u$ with width of the microchannel for the porousmedium model, computed at a constant mass flow rate of $0.5 \mathrm{~g} / \mathrm{s}$. The average Nusselt number values match the results from the unit-cell model to within $6 \%$. This further validates the porous-medium model. It may be mentioned here that the porous-medium model is based upon assumed values for porosity, permeability as well as interstitial heat transfer coefficient, representative of regular microchannels, wherein the flow enters normal to the cross-section of the microchannel and travels along its length. However, in MMC heat sinks, the flow enters in a direction normal to the top of the microchannel, undergoes a 90-degree turn at the inlet, travels through the length of the microchannel, again undergoes a 90-degree turn, and then exits through the manifold outlet. Due to this complex flow path which is not accounted for in the inputs to the model, we see a slight discrepancy in the output hydrodynamic and thermal performance of the porous-medium model. Further, the bottom solid substrate which is a part of the unit-cell model, is not included in the porous-medium model in order to reduce complexity. This also contributes to the discrepancy in thermal performance results, since any conduction heat loss through the bottom substrate has been neglected. However, since the variation in outputs obtained from the porous-medium model are within $8 \%$ of those obtained from the unit-cell model, these differences in model conditions are neglected in the rest of this analysis.

After validating and verifying both models, the unit-cell model is employed for assessing the effect of various input parameters on friction factor and Nusselt number. The model is then employed for uncertainty quantification and optimization. Optimization results obtained for the probabilistic optimization using the porous-medium model are also reported and compared with those from the unitcell model in section 4.5, so as to demonstrate the utility of the approach for performing a system-level optimization, as against the single unit cell discussed earlier.

\subsection{Effect of Parameters}

As a first step, the effect of microchannel width and depth and manifold depth on the outputs is assessed. Each input geometric parameter is individually varied and the outputs - the hydrodynamic and 
thermal performance of the heat sink - are studied. The non-dimensional Nusselt number, Reynolds number and friction factor for the different cases are defined as:

$$
\begin{aligned}
& N u=\frac{h D_{h}}{k_{f}} \\
& \mathrm{Re}=\frac{u D_{h}}{v} \\
& f=\frac{\Delta P\left(D_{h} / L\right)}{0.5 \rho u^{2}}
\end{aligned}
$$

where $u$ is the velocity at the inlet of the microchannel, $D_{h}$ the hydraulic diameter of the microchannel, and $L$ is the total length of flow through the channel. The hydraulic diameter of the microchannel is calculated as [14]:

$$
D_{h}=\frac{2 D_{c} W_{c}}{\left(D_{c}+W_{c}\right)}
$$

The effect of the aspect ratio of the microchannel $\left(W_{c} / D_{c}\right)$ and the manifold depth $\left(D_{m}\right)$ on the nondimensional outputs is shown in Figure 6, computed for a fixed inlet coolant mass flow rate of $0.5 \mathrm{~g} / \mathrm{s}$. It may be observed that as the channel aspect ratio increases, both $N u$ and $f R e$ increase. This may be attributed to the increase in the hydraulic diameter of the channel, which results in a higher Reynolds number. An increase in the manifold depth is seen to increase the value of $f R e$ due to increased pressure drop. However, as may be observed from Figure 6 (a), a change in the manifold depth does not have a significant effect on $\mathrm{Nu}$. This may be attributed to the definition of the heat transfer coefficient which uses the base area of the heat sink, and is therefore not significantly affected by the manifold depth. Hence, a smaller value of $D_{m}$ would lead to a better overall performance. For the optimization procedure in this study, $D_{m}$ is fixed at a small value of $100 \mu \mathrm{m}$, and is not included as an optimization parameter.

\subsection{Response Surfaces}

Representative response surfaces capturing the effects of the input parameters on the output parameters of interest - pressure drop and heat transfer coefficient - are shown in Figure 7 (a) and (b), respectively, for a fixed overall inlet mass flow rate of $0.5 \mathrm{~g} / \mathrm{s}$. With all other parameters remaining constant, a fixed overall inlet mass flow rate results in fixed flow speed at the inlet of each microchannel, independent of microchannel, for a base heat sink of fixed dimensions. The influence of individual input parameters, $W_{c}$ and $r$, computed at the mean values of the fixed input parameters, is also shown in the insets of Figure 7. As expected, the pressure drop decreases as the microchannel width increases due to 
the lower flow resistance. Similarly, as the manifold ratio $\left(r=L_{i n} / L_{\text {out }}\right)$ increases at a constant flow speed for each microchannel, $\Delta P$ increases due to the increased inlet area, leading to an increased contraction area ratio at the inlet. Similarly, the heat transfer coefficient $h$ decreases as the microchannel width increases. However, it is observed to have an optimum value relative to the manifold ratio $r$ which may be explained as follows. As the manifold ratio increases, the inlet length $L_{i n}$ increases while the manifold length $L_{m}$ decreases so as to keep the overall flow length $L$ constant. Hence, as the manifold ratio increases, there is an increase in mass flow rate at the inlet, leading to an increase in $h$. However, the decrease in length of manifold $L_{m}$ also leads to a reduction in area available for heat transfer, thereby leading to a reduction in the heat transfer coefficient. Owing to these competing factors, the heat transfer coefficient displays an optimum value relative to the manifold ratio, which for this case was found to be at approximately $r=3$.

\subsection{Uncertainty Quantification Results}

The first step in the solution procedure is to perform uncertainty quantification to study the variation of the outputs relative to uncertainties in the various input parameters. For the purpose of demonstration, without loss of generality, geometric parameters such as channel width $W_{c}$, channel depth $D_{c}$, manifold depth $D_{m}$, manifold inlet length $L_{i n}$ and manifold outlet length $L_{\text {out }}$ are assumed to be uniformly distributed random variables. The wide range of variation considered in the input parameters is summarized in Table 3. The uncertainty quantification is performed using the Smolyak sparse grid of second order. For this case of 5 uniformly distributed uncertain variables, 71 evaluations are necessary for constructing the response surface. All the simulations are performed at an overall inlet mass flow rate of $0.5 \mathrm{~g} / \mathrm{s}$ and temperature $T_{\text {in }}=300 \mathrm{~K}$. Once the explicit gPC representation of the response surface is obtained, 10,000 samples are randomly drawn to calculate the output response characteristics. The PDFs of the heat transfer coefficient and pressure drop obtained for the range of uncertain inputs considered are shown in Figure 8 (a) and (b), respectively. The corresponding mean and standard deviation of the outputs are computed and compared against the deterministic values obtained by fixing the uncertain variables at their mean values. The results of both the probabilistic and deterministic simulations are reported in Table 3. For the probabilistic runs, the mean values are reported along with the standard deviation $\sigma$.

Due to the wide range of variation of inputs under consideration, we obtain a widely spread-out PDF for the variation in outputs. Also, there is a significant difference between the mean values of pressure drop obtained from the UQ study and that from the deterministic study obtained by fixing the uncertain variables at their mean values. We also note the large observed standard deviations of the outputs, $h$ and $\Delta P$. This demonstrates the importance of using a probabilistic approach for design and optimization of MMC heat sinks. 
Apart from UQ, a sensitivity analysis is also performed employing DAKOTA. In order to assess the sensitivity of outputs to various inputs under consideration, uncertainty quantification analysis is performed by varying a single input parameter for which the sensitivity is being assessed, while keeping all the other inputs fixed at their mean values. The inputs relative to which a higher standard deviation is obtained in the outputs are identified as the more sensitive variables. The standard deviations of the outputs, heat transfer coefficient and pressure drop, obtained as the various input parameters are varied are listed in Table 4. Of all the variables considered, the outputs are most sensitive to variations in the width of the microchannel $W_{c}$ and the length of the inlet manifold $L_{i n}$. This information is valuable for design of experiments [16], since the primitive UQ results can be used to obtain an estimate of the most sensitive parameters, and the uncertainties in these parameters can then be resolved by a finer UQ analysis. Also, the insensitive parameters may be assumed as deterministic, thereby enhancing computational efficiency [16].

\subsection{Optimization}

The results of the deterministic and probabilistic optimization obtained by employing the unit-cell model are presented here, along with results of probabilistic optimization from the system-level porousmedium model.

A conventional, deterministic optimization study is first performed to arrive at the optimum geometry without considering the effect of uncertainties. In order to validate the optimization process, a simple case is considered. For this case, a single-objective optimization is performed, so as to find the optimum width for maximizing heat transfer coefficient at an overall inlet mass flow rate of $0.5 \mathrm{~g} / \mathrm{s}$. The mass flow rate at the inlet of the unit cell is calculated from the overall mass flow rate by considering the number of manifolds and microchannels appropriately. The microchannel width $W_{c}$ is allowed to take values between $10 \mu \mathrm{m}$ and $100 \mu \mathrm{m}$ for the optimization. Starting with an initial guess value of $W_{c}=80 \mu \mathrm{m}$, the optimum width of $W_{c}=10 \mu \mathrm{m}$ is predicted within about 7 iterations. As expected, the minimum microchannel width results in the maximum heat transfer coefficient and hence, the optimization process is validated.

As the mass flow rate at the inlet is increased, although the thermal performance improves, the pressure drop also increases significantly. Hence, a multi-objective optimization is performed. The following objective function that takes into account the effect of both $h$ and $\Delta P$ with appropriate scaling is considered:

$$
O F=w_{1}\left(h / h_{\max }\right)-w_{2}\left(\Delta P / \Delta P_{\max }\right)
$$

Here $h_{\max }$ and $\Delta P_{\max }$ are the maximum values of heat transfer coefficient and pressure drop, respectively, for the range of variation of inputs considered. This scaling of the outputs ensures that both thermal and 
flow characteristics are of the same order of magnitude, for comparison. Weight functions $w_{1}$ and $w_{2}$ sum up to a value of 1 , with their individual values depending on the relative importance ascribed to the two performance metrics. Thus, for an assumed set of weight functions, maximizing this objective function ensures an optimized geometry with maximum heat transfer coefficient and minimum pressure drop.

The geometric parameters of the manifold are taken into account by a non-dimensional manifold ratio $r$ given by:

$$
\begin{aligned}
& r=L_{\text {in }} / L_{\text {out }} \\
& L_{m}=L-L_{\text {in }}-L_{\text {out }}
\end{aligned}
$$

In this study, it may be noted that the length of the outlet manifold is fixed at $L_{\text {out }}=72 \mu \mathrm{m}$, while the length of the inlet manifold $L_{i n}$ is computed from Eq (18) for various values of $r$. Also, the total flow length is fixed at $L=400 \mu \mathrm{m}$, and the length of the manifold is computed from Eq (18), as indicated. The input geometric parameters considered for the optimization are the microchannel width $W_{c}$ and the manifold ratio $r$. Beginning with initial guess values, the optimizer iteratively varies the values of these variables until convergence is achieved. The optimization is performed for two different input conditions, $\dot{m}=0.5 \mathrm{~g} / \mathrm{s}$ and $\dot{m}=1.5 \mathrm{~g} / \mathrm{s}$, and for different weighting functions, $w_{1}=0.5, w_{2}=0.5$ and $w_{1}=0.7$ and $w_{2}$ $=0.3$, respectively.

Besides this deterministic optimization, we also perform a probabilistic optimization using the nested OUU approach described previously. The OUU is performed to predict the design variables that maximize the objective function, taking into account uncertainties in the geometric parameters, while also restricting the standard deviation of the objective function to a prescribed value, thus resulting in a robust design. In this case, the microchannel width $W_{c}$ and manifold ratio $r$ are considered as design variables with specified uncertainties. Thus, for each set of values of $W_{c}$ and $r$ obtained from the optimizer, the uncertainties are imposed in the inner UQ loop. This is achieved by appropriate mapping of the outer loop variables into the inner loop [28]. The depth of the microchannel $D_{c}$ is also considered as an uncertain variable with specified uncertainty. From Eq (18), the uncertainty in manifold ratio translates to uncertainties in the manifold lengths $L_{i n}$ and $L_{m}$. Table 5 lists the values of the normal uncertain variables with the standard deviation considered for this analysis. It may be noted that the uncertainties considered in the present study are based on approximate tolerances specified by the manufacturers. The output - the scaled heat transfer coefficient ratio - is subjected to a constraint, bounding its standard deviation. The OUU problem statement is formally defined as: 


$$
\begin{array}{ccc}
\text { Maximize } & O F=w_{1}\left(h / h_{\max }\right)-w_{2}\left(\Delta P / \Delta P_{\max }\right) \\
\text { such that } & \sigma\left(h / h_{\max }\right) \leq 0.02, \quad \dot{m}=0.5 g / s \\
& \sigma\left(h / h_{\max }\right) \leq 0.035, \quad \dot{m}=1.5 g / s
\end{array}
$$

Figure 9 shows the convergence history of the optimization procedure for two representative cases with Figure 9 (a) corresponding to deterministic optimization with $\dot{m}=1.5 \mathrm{~g} / \mathrm{s}$ and $w_{1}=w_{2}=0.5$, and Figure 9 (b) to probabilistic optimization with $\dot{m}=1.5 \mathrm{~g} / \mathrm{s}, w_{1}=0.7$ and $w_{2}=0.3$. For the case of probabilistic optimization, the variation in standard deviation of the output $\left(h / h_{\max }\right)$ is also shown. For the different input conditions and weighting functions considered in this work, the corresponding converged values of the design parameters are shown in Table 6 for deterministic as well as probabilistic optimization cases, with the first column describing the condition for which the optimization is performed, i.e, the input mass flow rate, the objective function weights and the values of $h_{\max }$ and $\Delta P_{\max }$. It may be observed from Table 6 that for both deterministic and probabilistic optimization, as the value of $w_{l}$ is increased, the value of optimum width decreases and that of manifold ratio increases. This is due to the fact that a higher value of $w_{l}$ means that the objective function is dominated by the heat transfer coefficient, the value of which increases as the width decreases and manifold ratio increases. Also, for the case of probabilistic optimization, the imposed constraint restricting the standard deviation of the scaled output results in more conservative values for the geometric parameters, as also observed by Bodla et al. [15] for the case of pin-fin heat sinks. Hence, the use of this approach allows us to quantify precisely how conservative the design needs to be in order to account for the uncertainties. Furthermore, owing to the conservative nature of the design, the output objective function is lower for the probabilistic case than that obtained with the deterministic counterpart. At the same time, by accounting for uncertainties as part of the optimization procedure, the probabilistic design ensures a more predictable and robust design. The convergence history shown in Figure 9 (a) and (b) may also be used to gain a first estimate of the expected value of the output when values for the design variables other than the final converged value are chosen. Such history data may also be used to assess whether a tighter or looser convergence criterion may be employed for obtaining better converged results quickly [15].

System-level optimization under uncertainty is demonstrated with an OUU study employing the porous-medium model. The analysis considers the same set of input parameter variations and constraints and an inlet mass flow rate of $0.5 \mathrm{~g} / \mathrm{s}$ as used above with the unit-cell model. The results obtained from the porous-medium model are shown in Table 7 along with those from the unit-cell model. It may be observed that the optimum microchannel width and manifold inlet length obtained with both models are in close agreement with each other. This further validates the porous-medium model and demonstrates its utility for performing a system-level optimization analysis. Such a model may be used for analysis of 
complex, realistic cases such as those involving non-uniform heat fluxes. As described in section 4.1, the optimum mesh size required for the porous-medium model for the entire manifold length ( 25 unit cells) is about half that of the unit-cell model for a single microchannel, which results in a corresponding reduction in computational time for the porous-medium model.

The OUU analysis in this study is performed using a Smolyak sparse grid of level 1, which results in 7 inner loop evaluations for each outer loop evaluation, for the case of 3 uncertain variables. A complete OUU evaluation converges in approximately 20 outer loop evaluations. A complete optimization study in this case therefore requires 140 overall evaluations. Using the parallelized CFD capabilities of FLUENT, and available computational resources, i.e., Quad-Core Intel E5410 processors, all the inner loop evaluations were run in parallel, so that each outer loop evaluation of the unit-cell model required roughly 90 minutes of real time. Similarly, each outer loop evaluation employing the porous-medium model required around 45 minutes. Thus, the total time required for one complete probabilistic optimization study was approximately 30 and 15 hours for the unit-cell (single channel) and porous-medium (multiple channels) models, respectively. The porous-medium model offers a cost-effective, alternative approach that is useful for system-level optimizations.

\section{Conclusions}

A 3-D numerical model for manifold microchannel (MMC) heat sinks is developed and validated. Further, an Uncertainty Quantification (UQ) analysis is performed to demonstrate the effect of input uncertainties on the output parameters of interest. A cumulative objective function is defined for considering the two outputs of interest in the design of heat sinks, i.e., heat transfer coefficient and pressure drop. A unit-cell geometry of the MMC heat sink is optimized by taking into account the effect of inherent uncertainties present in the various input parameters. A framework for performing such probabilistic optimization is developed in DAKOTA, an open-source optimization and uncertainty quantification toolkit. The corresponding results obtained via the Optimization Under Uncertainty (OUU) approach are compared with those obtained with a conventional deterministic counterpart, and the conservative nature of the probabilistic design approach is quantified. Based on sensitivity information, the critical input parameters to which the output quantities are most sensitive are also identified. In addition, a cost-effective porous-medium model for the MMC heat sinks is presented and validated, and subsequently used for optimization under uncertainty. This model provides a system-level optimization of the geometry taking all the microchannels in the heat sink into account, as against a single microchannel considered in the unit-cell approach, thereby allowing the designer to consider complex, realistic cases of non-uniformly applied heat fluxes. A representative case is considered and the utility of the model is demonstrated by comparing against the detailed unit-cell model. 


\section{Acknowledgements}

The authors acknowledge support for this work from industry members of the Cooling Technologies Research Center, an NSF Industry/University Cooperative Research Center. 


\section{References}

[1] D.B. Tuckerman and R.F.W. Pease, High-performance heat sinking for VLSI, IEEE Electron Device Letters 2 (1981) 126-129.

[2] S.V. Garimella and T. Harirchian, Microchannel Heat Sinks for Electronics Cooling, Vol. 1 in the Encyclopedia of Thermal Packaging, World Scientific, Singapore, 2013, pp. 248.

[3] K. Vafai and L. Zhu, Analysis of two-layered microchannel heat sink concept in electronic cooling, International Journal of Heat and Mass Transfer 42 (1999) 2287-2297.

[4] J.H. Ryu, D.H. Choi, and S.J. Kim, Numerical optimization of the thermal performance of a microchannel heat sink, International Journal of Heat and Mass Transfer 45 (2002) 2823-2827.

[5] P. S. Lee and S. V. Garimella, Thermally developing flow and heat transfer in rectangular microchannels of different aspect ratios, International Journal of Heat and Mass Transfer 49 (17) (2006) 3060-3067.

[6] T. C. Hung, W. M. Yan, X. D. Wang, and Y. X. Huang, Optimal design of geometric parameters of double-layered microchannel heat sinks, International Journal of Heat and Mass Transfer 55 (2012) 3262-3272.

[7] T. C. Hung and W. M. Yan, Optimization of a microchannel heat sink with varying channel heights and widths, Numerical Heat Transfer-Part A 62 (2012) 722-741.

[8] G. M. Harpole and J. E. Eninger, Micro-channel heat exchanger optimization, in Proceedings of the 7th IEEE Semiconductor Thermal Measurement and Management Symposium, 1991, pp. 59-63.

[9] E. Kermani, Manifold micro-channel cooling of photovoltaic cells for high efficiency solar energy conversion, M.S. Thesis, University of Maryland, 2008.

[10] E. Kermani, S. Dessiatoun, A. Shooshtari, and M. M. Ohadi, Experimental Investigation of Heat Transfer Performance of a Manifold Microchannel Heat Sink for Cooling of Concentrated Solar Cells, Proceedings of the 59th Electronic Components and Technology Conference, 2009, pp. 453459.

[11] D. Copeland, H. Takihara, and W. Nakayama, Manifold microchannel heat sinks: theory and experiments, Thermal Science and Engineering 3 (2) (1995) 9-15.

[12] Y.I. Kim, W.C. Chun, J.T. Kim, B.C. Pak, and B.J. Baek, Forced air cooling by using manifold microchannel heat sinks, KSME International Journal 12 (4) (1998) 709-718.

[13] D. Copeland, M. Behnia, and W. Nakayama, Manifold microchannel heat sinks: isothermal analysis, IEEE Transactions on Components, Packaging, and Manufacturing Technology: Part A 20 (1997) 96-102.

[14] J. Ryu, D. Choi, and S. Kim, Three-dimensional numerical optimization of a manifold microchannel heat sink, International Journal of Heat and Mass Transfer 46 (2003) 1553-1562.

[15] K. K. Bodla, J. Y. Murthy, and S. V. Garimella, Optimization under uncertainty applied to heat sink design, ASME Journal of Heat Transfer 135 (2013).

[16] M. S. Eldred, A. A. Giunta, S. F. Wojtkiewicz Jr, and T. G. Trucano, Formulations for surrogatebased optimization under uncertainty, in Proceedings of the 9th AIAA/ISSMO Symposium on Multidisciplinary Analysis and Optimization, 2002, pp. 4-6.

[17] D. Liu and Garimella S.V., Analysis and Optimization of the Thermal Performance of Microchannel Heat Sinks, International Journal of Numerical Methods for Heat and Fluid Flow 15 (1) (2004) 7 26.

[18] D. Xiu and G. E. Karniadakis, The Wiener-Askey polynomial chaos for stochastic differential equations, SIAM Journal on Scientific Computing 24 (2) (2002) 619-44.

[19] F. P. Incropera and D. P. De Witt, Fundamentals of Heat and Mass Transfer, Singapore: John Wiley \& Sons (Asia).

[20] W. Escher, B. Michel, and D. Poulikakos, A novel high performance, ultra thin heat sink for electronics, International Journal of Heat and Fluid Flow 31 (4) (2010) 586-598.

[21] T. CUBIT, CUBIT 10.0 User's Manual, Sandia National Laboratories, Albuquerque, NM, 2005. 
[22] User's Guide for ANSYS FLUENT 14.0. Fluent Inc., 2011.

[23] Kaviany M., Principles of Heat Transfer in Porous Media, 1st ed. New York: Springer-Verlag, 1991.

[24] Kim S.J. and Kim D., Forced convection in microstructure for electronic equipment cooling 121 (1999) 639-45.

[25] M. S. Eldred, Recent advances in non-intrusive polynomial chaos and stochastic collocation methods for uncertainty analysis and design, in 50th AIAA/ASME/ASCE/AHS/ASC Structures, Structural Dynamics and Materials Conference, Palm Springs, CA, United States, May 2009.

[26] I. M. Sobol and S. Kucherenko, Derivative based global sensitivity measures and their link with global sensitivity indices, Mathematics and Computers in Simulation 79 (10) (2009) 3009-3017.

[27] M. S. Eldred, A. A. Giunta, B. G. van Bloemen Waanders, S. F. Wojtkiewicz, W. E. Hart, and M. P. Alleva, DAKOTA, a multilevel parallel object-oriented framework for design optimization, parameter estimation, uncertainty quantification, and sensitivity analysis: version 4.1 reference manual, Sandia National Laboratories Albuquerque, NM, 2007.

[28] G. N. Vanderplaats, CONMIN, a FORTRAN program for constrained function minimization: user's manual 62282. Ames Research Center and US Army Air Mobility R\&D Laboratory, 1973. 


\section{List of Tables}

Table 1: Effect of mass flow rate and comparison of hydrodynamic performance of MMC heat sink, as predicted by the unit-cell and porous-medium models. The present case corresponds to $W_{c}=20 \mu \mathrm{m}, D_{c}=$ $200 \mu \mathrm{m}, D_{m}=500 \mu \mathrm{m}, L_{\text {in }}=60 \mu \mathrm{m}$, and $L_{\text {out }}=120 \mu \mathrm{m}$.

Table 2: Effect of channel width and comparison of (a) hydrodynamic and (b) thermal performance, as predicted by the unit-cell and porous-medium models. The present case corresponds to a fixed mass flow rate of $0.5 \mathrm{~g} / \mathrm{s}$.

Table 3: Uncertainty quantification for MMC heat sinks.

Table 4: Sensitivity analysis for MMC heat sinks.

Table 5: Input parameters for deterministic and probabilistic optimization of an MMC heat sink: $W_{c \text {, det }}$ and $r_{d e t}$ are the mean values of the variables obtained from each iteration of the outer optimization loop. Table 6: Comparison of deterministic and probabilistic optimization results for different mass flow rates and weighing functions.

Table 7: Comparison of OUU results obtained via the unit-cell and porous-medium models. The predicted geometry is found to match reasonably well. 


\section{List of Figures}

Figure 1: Computational domains for the MMC heat sink: (a) complete heat sink with coolant path, (b) unit-cell model used for direct simulation, showing geometric parameters and boundary conditions and (c) computational domain for the porous-medium model along with boundary conditions.

Figure 2: OUU approach employed in the present work, adapted from [15].

Figure 3: Mesh-independence study, performed for an overall inlet mass flow rate of $0.5 \mathrm{~g} / \mathrm{s}$ : (a) unit-cell model with channel dimensions $W_{c}=80 \mu \mathrm{m}, D_{c}=200 \mu \mathrm{m}, D_{m}=300 \mu \mathrm{m}, L_{\text {out }}=120 \mu \mathrm{m}, r=0.5$, and $L=$ $160 \mu \mathrm{m}$, and (b) porous-medium model with channel dimensions $W_{c}=80 \mu \mathrm{m}, D_{c}=200 \mu \mathrm{m}, D_{m}=300$ $\mu \mathrm{m}, L_{\text {out }}=72 \mu \mathrm{m}, r=4.0$, and $L=1000 \mu \mathrm{m}$. The optimum mesh size for which the pressure drop matches to within $0.3 \%$ of the value with the finest mesh size considered is highlighted.

Figure 4: Velocity vectors and temperature contours for channel dimensions $\mathrm{W}_{\mathrm{c}}=40 \mu \mathrm{m}, \mathrm{D}_{\mathrm{c}}=200 \mu \mathrm{m}$, $\mathrm{D}_{\mathrm{m}}=500 \mu \mathrm{m}, \mathrm{L}_{\text {in }}=60 \mu \mathrm{m}, \mathrm{L}_{\text {out }}=120 \mu \mathrm{m}$ : (a) Velocity vectors at center plane of microchannel (plane shown in red dashed lines in the inset) for overall inlet mass flow rate $=0.5 \mathrm{~g} / \mathrm{s}$; (b) Temperature contours at center plane of microchannel for overall inlet mass flow rate of $0.5 \mathrm{~g} / \mathrm{s}$; and (c) Temperature contours at center plane of microchannel for overall inlet mass flow rate of $5.0 \mathrm{~g} / \mathrm{s}$. Velocity values are in $\mathrm{m} / \mathrm{s}$ and temperature values are in Kelvin.

Figure 5: Heat transfer coefficient as a function of flow rate for a channel aspect ratio $=0.1$. Also shown are experimental results along with reported uncertainties from Kermani [9].

Figure 6: Effect of the geometric parameters on outputs: (a) $\mathrm{Nu}$, and (b) fRe, computed for the case of fixed coolant mass flow rate of $0.5 \mathrm{~g} / \mathrm{s}$.

Figure 7: Representative response surfaces of (a) pressure drop, and (b) heat transfer coefficient, shown as a function of variation in channel width and manifold ratio. The insets show variation of the outputs relative to variation in each input parameter, obtained by holding the other input parameter at its mean value as indicated.

Figure 8: PDF of (a) heat transfer coefficient, and (b) pressure drop for uniformly distributed input parameters in Table 3.

Figure 9: Convergence history of (a) deterministic optimization for $w_{1}=w_{2}=0.5$, and (b) probabilistic optimization for $w_{1}=0.7$ and $w_{2}=0.3$. 
Table 1: Effect of mass flow rate and comparison of hydrodynamic performance of MMC heat sink, as predicted by the unit-cell and porous-medium models. The present case corresponds to $W_{c}=20 \mu \mathrm{m}, D_{c}=$ $200 \mu \mathrm{m}, D_{m}=500 \mu \mathrm{m}, L_{\text {in }}=60 \mu \mathrm{m}$, and $L_{\text {out }}=120 \mu \mathrm{m}$.

\begin{tabular}{cccc}
\hline $\begin{array}{c}\text { Mass Flow Rate } \\
(\mathbf{g} / \mathbf{s})\end{array}$ & $\begin{array}{c}\Delta \mathbf{P}(\mathbf{P a}) \\
\text { Unit-Cell Model }\end{array}$ & $\begin{array}{c}\Delta \mathbf{P}(\mathbf{P a}) \\
\text { Porous-Medium Model }\end{array}$ & $\begin{array}{c}\text { Difference } \\
( \pm)\end{array}$ \\
\hline $\mathbf{0 . 5}$ & 352.7 & 331.1 & 6.1 \\
\hline $\mathbf{1 . 0}$ & 693.5 & 675.3 & 2.6 \\
\hline $\mathbf{1 . 5}$ & 1041.3 & 1032.6 & 0.8 \\
\hline $\mathbf{2 . 0}$ & 1390.6 & 1403.1 & 0.9 \\
\hline $\mathbf{2 . 5}$ & 1741.6 & 1786.7 & 2.6 \\
\hline
\end{tabular}


Table 2: Effect of channel width and comparison of (a) hydrodynamic and (b) thermal performance, as predicted by the unit-cell and porous-medium models. The present case corresponds to a fixed mass flow rate of $0.5 \mathrm{~g} / \mathrm{s}$.

(a)

\begin{tabular}{cccc|ccc}
\hline $\begin{array}{c}\text { Channel } \\
\text { Width } \\
\boldsymbol{W}_{\boldsymbol{c}}(\boldsymbol{\mu \mathrm { m }})\end{array}$ & $\begin{array}{c}\Delta \mathbf{P}(\mathbf{P a}) \\
\text { Unit-Cell } \\
\text { Model }\end{array}$ & $\begin{array}{c}\Delta \mathbf{P}(\mathbf{P a}) \\
\text { Porous-Medium } \\
\text { Model }\end{array}$ & $\begin{array}{c}\text { Difference } \\
( \pm \%)\end{array}$ & $\begin{array}{c}\text { Nu } \\
\text { Unit-Cell } \\
\text { Model }\end{array}$ & $\begin{array}{c}\text { Nu } \\
\text { Porous-Medium } \\
\text { Model }\end{array}$ & $\begin{array}{c}\text { Difference } \\
( \pm \%)\end{array}$ \\
\hline $\mathbf{2 0}$ & 341.3 & 315.2 & 7.6 & 2.10 & 2.11 & 0.4 \\
\hline $\mathbf{4 0}$ & 106.1 & 98.4 & 7.2 & 3.87 & 3.79 & 2.1 \\
\hline $\mathbf{6 0}$ & 58.7 & 55.3 & 5.8 & 5.32 & 5.05 & 5.0 \\
\hline $\mathbf{8 0}$ & 41.1 & 39.9 & 2.9 & 6.39 & 6.02 & 5.8 \\
\hline
\end{tabular}


Table 3: Uncertainty quantification for MMC heat sinks.

\begin{tabular}{ccc}
\hline Parameter & $\begin{array}{c}\text { Deterministic } \\
\text { approach }\end{array}$ & $\begin{array}{c}\text { Probabilistic approach, } \\
\text { Uniform random } \\
\text { distribution of inputs }\end{array}$ \\
\hline Inputs & 200 & $\begin{array}{c}\text { Minimum }=100 \\
\text { Maximum }=300\end{array}$ \\
\hline$D_{c}(\mu \mathrm{m})$ & 260 & $\begin{array}{c}\text { Minimum }=20 \\
\text { Maximum }=500\end{array}$ \\
\hline$W_{c}(\mu \mathrm{m})$ & 260 & $\begin{array}{c}\text { Minimum }=100 \\
\text { Maximum }=300\end{array}$ \\
\hline$D_{m}(\mu \mathrm{m})$ & 60 & $\begin{array}{c}\text { Minimum }=20 \\
\text { Maximum }=200\end{array}$ \\
\hline$L_{\text {in }}(\mu \mathrm{m})$ & 120 & $\begin{array}{c}\text { Minimum }=20 \\
\text { Maximum }=200\end{array}$ \\
\hline$L_{\text {out }}(\mu \mathrm{m})$ & & $\begin{array}{c}\text { Mean }=30120 \\
\sigma=10703\end{array}$ \\
\hline Outputs & 29120 & $\begin{array}{c}\text { Mean }=56 \\
\sigma=27\end{array}$ \\
\hline$h\left(\mathrm{~W} / \mathrm{m}^{2} \mathrm{~K}\right)$ & 32 &
\end{tabular}


Table 4: Sensitivity analysis for MMC heat sinks.

\begin{tabular}{|c|c|c|c|}
\hline $\begin{array}{l}\text { Variable } \\
\text { input }\end{array}$ & Range of variation in input & $\begin{array}{c}\text { Std. deviation in } \\
\mathbf{h}\left(W / \mathbf{m}^{2} \mathbf{K}\right)\end{array}$ & $\begin{array}{l}\text { Std. deviation } \\
\text { in } \Delta P(\mathbf{P a})\end{array}$ \\
\hline$W_{c}(\mu \mathrm{m})$ & $\begin{array}{c}\text { Uniform random, } \\
\min .=40 \\
\max .=80\end{array}$ & 419 & 23.7 \\
\hline$L_{i n}(\mu \mathrm{m})$ & $\begin{array}{c}\text { Uniform random, } \\
\text { min. }=50 \\
\max =80\end{array}$ & 5325 & 2.7 \\
\hline$D_{c}(\mu \mathrm{m})$ & $\begin{array}{c}\text { Uniform random, } \\
\min .=180 \\
\max .=220\end{array}$ & 234 & 1.2 \\
\hline$L_{\text {out }}(\mu \mathrm{m})$ & $\begin{array}{c}\text { Uniform random, } \\
\min .=100 \\
\max .=140\end{array}$ & 35 & 2.7 \\
\hline
\end{tabular}


Table 5: Input parameters for deterministic and probabilistic optimization of an MMC heat sink: $W_{c, \text { det }}$ and $r_{d e t}$ are the mean values of the variables obtained from each iteration of the outer optimization loop.

\begin{tabular}{|c|c|c|}
\hline Parameter & $\begin{array}{l}\text { Deterministic } \\
\text { optimization }\end{array}$ & $\begin{array}{l}\text { Probabilistic } \\
\text { optimization }\end{array}$ \\
\hline$L_{\text {out }}(\mu \mathrm{m})$ & 72 & 72 \\
\hline$D_{c}(\mu \mathrm{m})$ & 200 & $\begin{array}{c}\text { Mean }=200 \\
\sigma=10\end{array}$ \\
\hline$W_{c}(\mu \mathrm{m})$ & $\begin{aligned} \text { Minimum } & =10 \\
\text { Maximum } & =100\end{aligned}$ & $\begin{array}{c}\text { Minimum }=10 \\
\text { Maximum }=100 \\
\text { Mean }=W_{c, d e t} \\
\sigma=10\end{array}$ \\
\hline$r$ & $\begin{array}{l}\text { Minimum }=0.5 \\
\text { Maximum }=4\end{array}$ & $\begin{array}{c}\text { Minimum }=0.5 \\
\text { Maximum }=4 \\
\text { Mean }=r_{d e t} \\
\sigma=0.1\end{array}$ \\
\hline$L_{i n}(\mu \mathrm{m})$ & $\begin{array}{c}\text { Minimum }=36 \\
\text { Maximum }=288\end{array}$ & $\begin{array}{c}\text { Minimum }=36 \\
\text { Maximum }=288 \\
\sigma=7.2\end{array}$ \\
\hline$L_{m}(\mu \mathrm{m})$ & $\begin{array}{c}\text { Minimum }=40 \\
\text { Maximum }=292\end{array}$ & $\begin{array}{c}\text { Minimum }=40 \\
\text { Maximum }=292 \\
\sigma=7.2\end{array}$ \\
\hline
\end{tabular}


Table 6: Comparison of deterministic and probabilistic optimization results for different mass flow rates and weighing functions.

(a) $\dot{m}=0.5 \mathrm{~g} / \mathrm{s}$

\begin{tabular}{|c|c|c|c|}
\hline Condition & Parameter & $\begin{array}{l}\text { Deterministic } \\
\text { optimization }\end{array}$ & $\begin{array}{c}\text { Probabilistic } \\
\text { optimization } \\
\text { (Mean values) } \\
\end{array}$ \\
\hline \multirow{4}{*}{$\begin{array}{c}\dot{m}=0.5 \mathrm{~g} / \mathrm{s} \\
h_{\max }=120,000 \mathrm{~W} / \mathrm{m}^{2} \mathrm{~K} \\
\Delta P_{\max }=3000 \mathrm{~Pa}\end{array}$} & \multicolumn{3}{|l|}{ Inputs } \\
\hline & $W_{c}(\mu \mathrm{m})$ & 33.3 & 26.5 \\
\hline & $r$ & 3.25 & 2.5 \\
\hline & $L_{i n}(\mu \mathrm{m})$ & 234 & 180 \\
\hline \multirow{5}{*}{$\begin{array}{c}w_{1}=0.5 \\
w_{2}=0.5 \\
O F=0.5\left(h / h_{\max }\right)- \\
0.5\left(\Delta P / \Delta P_{\max }\right)\end{array}$} & $L_{m}(\mu \mathrm{m})$ & 94 & 148 \\
\hline & \multicolumn{3}{|l|}{ Outputs } \\
\hline & $h\left(\mathrm{~W} / \mathrm{m}^{2} \mathrm{~K}\right)$ & 92185 & 85,766 \\
\hline & $\Delta P(\mathrm{~Pa})$ & 382 & 532 \\
\hline & $O F$ & 0.3204 & 0.2686 \\
\hline \multirow{6}{*}{$\begin{array}{c}\dot{m}=0.5 \mathrm{~g} / \mathrm{s} \\
h_{\max }=120,000 \mathrm{~W} / \mathrm{m}^{2} \mathrm{~K} \\
\Delta P_{\max }=3000 \mathrm{~Pa}\end{array}$} & \multicolumn{3}{|l|}{ Inputs } \\
\hline & $W_{c}(\mu \mathrm{m})$ & 27.6 & 24.2 \\
\hline & $r$ & 3.4 & 2.9 \\
\hline & $L_{i n}(\mu \mathrm{m})$ & 245 & 209 \\
\hline & $L_{m}(\mu \mathrm{m})$ & 83 & 119 \\
\hline & \multicolumn{3}{|l|}{ Outputs } \\
\hline \multirow{3}{*}{$\begin{array}{c}O F=0.7\left(h / h_{\max }\right)- \\
0.3\left(\Delta P / \Delta P_{\max }\right)\end{array}$} & $h\left(\mathrm{~W} / \mathrm{m}^{2} \mathrm{~K}\right)$ & 95940 & 93,257 \\
\hline & $\Delta P(\mathrm{~Pa})$ & 520 & 689 \\
\hline & $O F$ & 0.5076 & 0.475 \\
\hline
\end{tabular}


(b) $\quad \dot{m}=1.5 \mathrm{~g} / \mathrm{s}$

\begin{tabular}{|c|c|c|c|}
\hline Condition & Parameter & Deterministic optimization & $\begin{array}{l}\text { Probabilistic } \\
\text { optimization }\end{array}$ \\
\hline \multirow{4}{*}{$\begin{array}{c}\dot{m}=1.5 \mathrm{~g} / \mathrm{s} \\
h_{\max }=180000 \mathrm{~W} / \mathrm{m}^{2} \mathrm{~K} \\
\Delta P_{\max }=10000 \mathrm{~Pa}\end{array}$} & Inputs & & \\
\hline & $W_{c}(\mu \mathrm{m})$ & 27 & 25.6 \\
\hline & $r$ & 2.47 & 1.8 \\
\hline & $L_{\text {in }}(\mu \mathrm{m})$ & 178 & 115.2 \\
\hline \multirow{2}{*}{$\begin{array}{l}w_{1}=0.5 \\
w_{2}=0.5\end{array}$} & $L_{m}(\mu \mathrm{m})$ & 150 & 212.8 \\
\hline & Outputs & & \\
\hline \multirow{3}{*}{$\begin{array}{c}O F=0.5\left(h / h_{\max }\right)- \\
0.5\left(\Delta P / \Delta P_{\max }\right)\end{array}$} & $h\left(\mathrm{~W} / \mathrm{m}^{2} \mathrm{~K}\right)$ & 148314 & 123964 \\
\hline & $\Delta P(\mathrm{~Pa})$ & 1565 & 836 \\
\hline & $O F$ & 0.3337 & 0.3025 \\
\hline \multirow{3}{*}{$\begin{array}{c}\dot{m}=1.5 \mathrm{~g} / \mathrm{s} \\
h_{\max }=180000 \\
W / m^{2} \mathrm{~K}\end{array}$} & Inputs & & \\
\hline & $W_{c}(\mu \mathrm{m})$ & 22 & 21.3 \\
\hline & $r$ & 2.76 & 2.4 \\
\hline \multirow{2}{*}{$\Delta P_{\max }=10000 \mathrm{~Pa}$} & $L_{i n}(\mu \mathrm{m})$ & 199 & 173 \\
\hline & $L_{m}(\mu \mathrm{m})$ & 129 & 155 \\
\hline \multirow{2}{*}{$\begin{array}{l}w_{1}=0.7 \\
w_{2}=0.3\end{array}$} & Outputs & & \\
\hline & $h\left(\mathrm{~W} / \mathrm{m}^{2} \mathrm{~K}\right)$ & 156832 & 154511 \\
\hline \multirow{2}{*}{$\begin{array}{c}O F=0.7\left(h / h_{\max }\right)- \\
0.3\left(\Delta P / \Delta P_{\max }\right)\end{array}$} & $\Delta \mathrm{P}(\mathrm{Pa})$ & 2283 & 2567 \\
\hline & $O F$ & 0.5414 & 0.5238 \\
\hline
\end{tabular}


Table 7: Comparison of OUU results obtained via the unit-cell and porous-medium models. The predicted geometry is found to match reasonably well.

\begin{tabular}{ccccc}
\hline Condition & \multicolumn{2}{c}{ Unit-Cell Model } & \multicolumn{2}{c}{ Porous-Medium Model } \\
\hline $\mathbf{u}=\mathbf{0 . 0 3} \mathbf{~ m} / \mathbf{s}$ & $\mathrm{W}_{\mathrm{c}}(\mu \mathrm{m})$ & $\mathrm{L}_{\mathrm{in}}(\mu \mathrm{m})$ & $\mathrm{W}_{\mathrm{c}}(\mu \mathrm{m})$ & $\mathrm{L}_{\mathrm{in}}(\mu \mathrm{m})$ \\
\hline $\mathbf{0 . 7}\left(\mathbf{h} / \mathbf{h}_{\max }\right)+\mathbf{0 . 3}\left(-\Delta \mathbf{P} / \Delta \mathbf{P}_{\max }\right)$ & 24 & 209 & 30 & 212 \\
\hline $\mathbf{0 . 5}\left(\mathbf{h} / \mathbf{h}_{\max }\right)+\mathbf{0 . 5}\left(-\Delta \mathbf{P} / \Delta \mathbf{P}_{\max }\right)$ & 27 & 180 & 32 & 184 \\
\hline
\end{tabular}




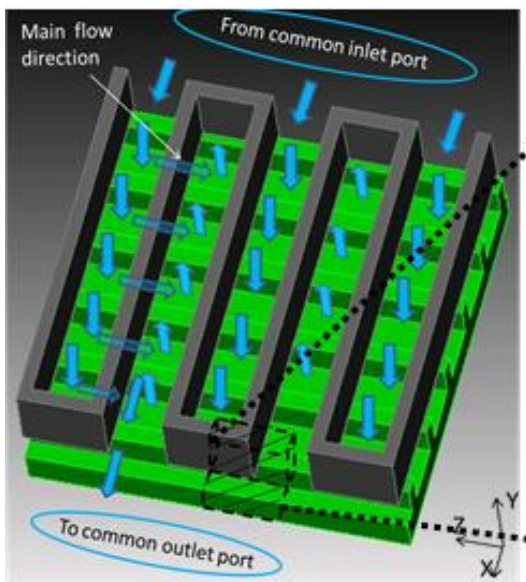

(a)

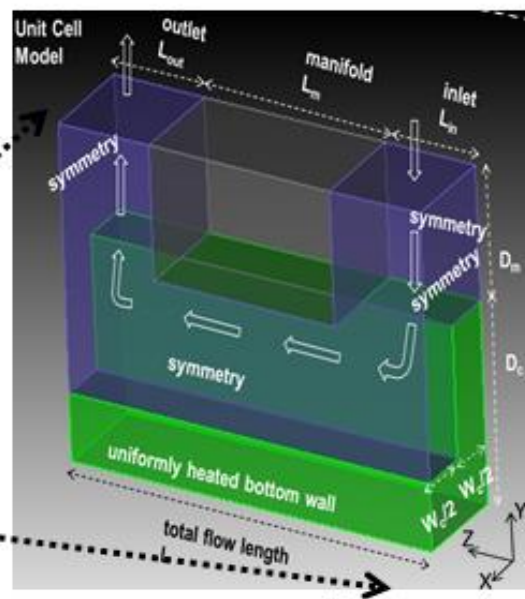

(b)

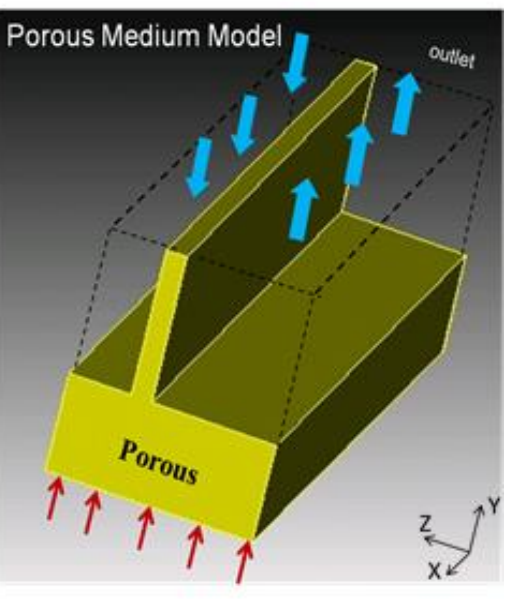

(c)

Figure 1: Computational domains for the MMC heat sink: (a) complete heat sink with coolant path, (b) unit-cell model used for direct simulation, showing geometric parameters and boundary conditions and (c) computational domain for the porous-medium model along with boundary conditions. 


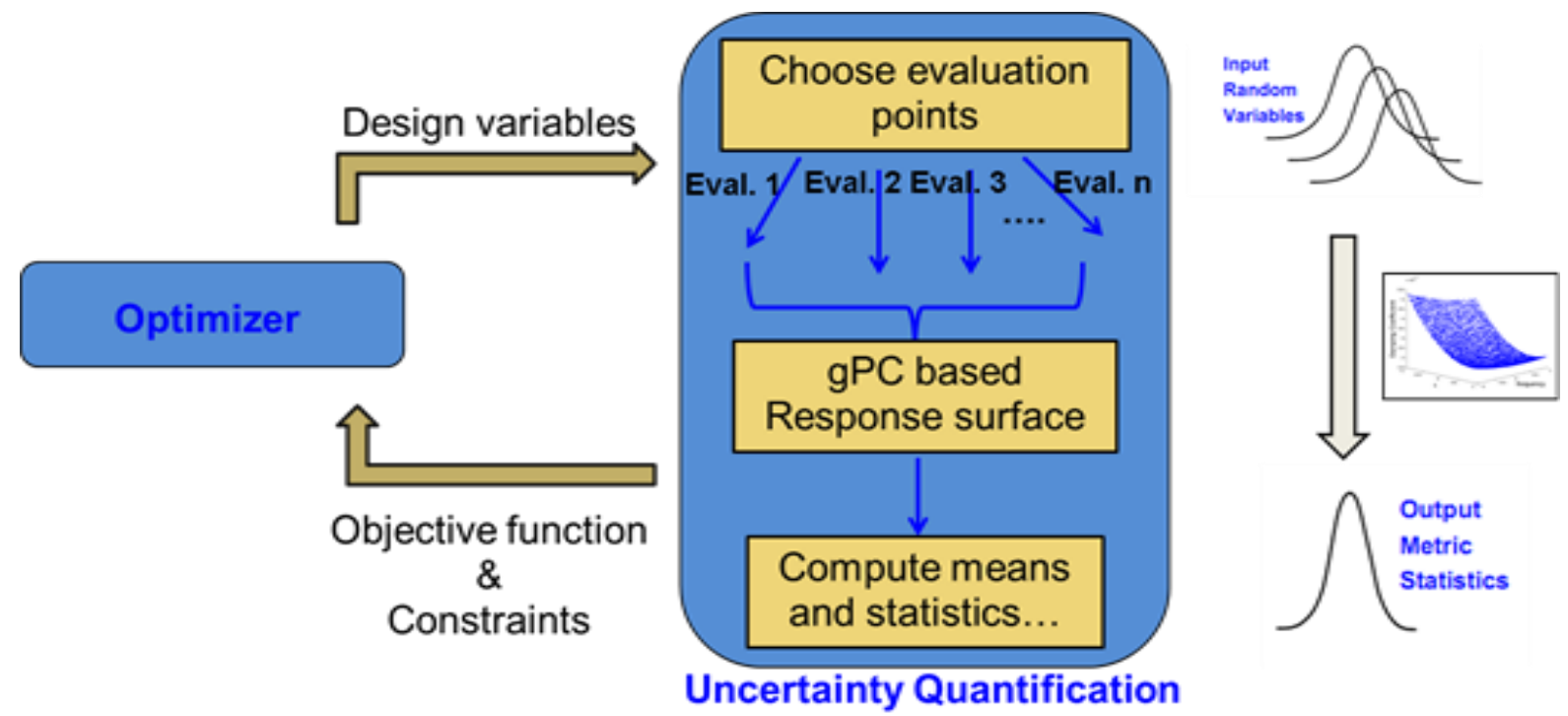

Figure 2: OUU approach employed in the present work, adapted from [15]. 


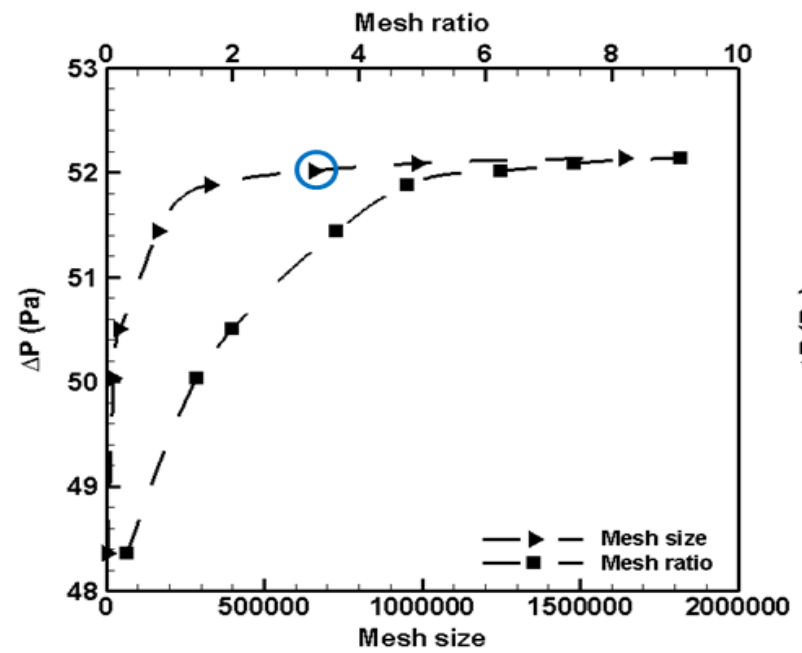

(a)

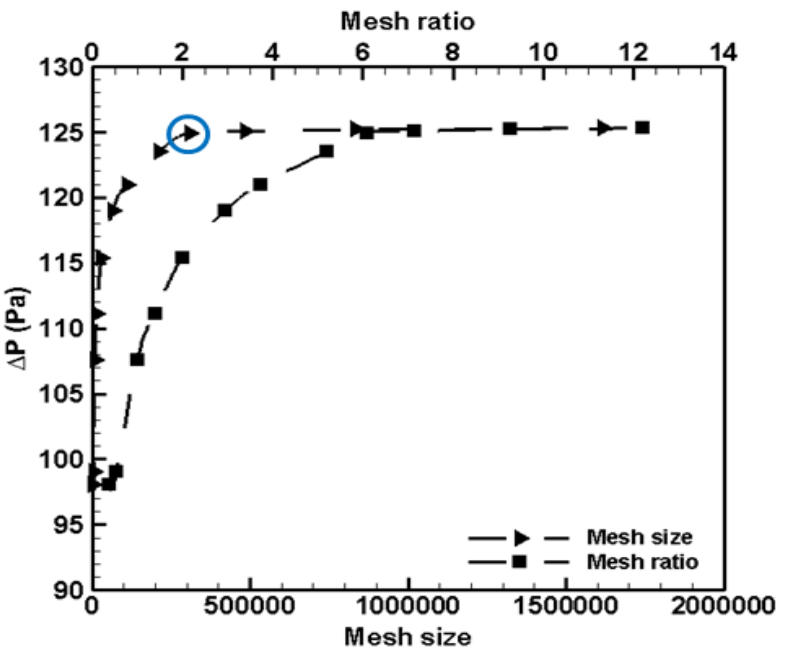

(b)

Figure 3: Mesh-independence study, performed for an overall inlet mass flow rate of $0.5 \mathrm{~g} / \mathrm{s}$ : (a) unit-cell model with channel dimensions $W_{c}=80 \mu \mathrm{m}, D_{c}=200 \mu \mathrm{m}, D_{m}=300 \mu \mathrm{m}, L_{\text {out }}=120 \mu \mathrm{m}, r=0.5$, and $L=$ $160 \mu \mathrm{m}$, and (b) porous-medium model with channel dimensions $W_{c}=80 \mu \mathrm{m}, D_{c}=200 \mu \mathrm{m}, D_{m}=300$ $\mu \mathrm{m}, L_{\text {out }}=72 \mu \mathrm{m}, r=4.0$, and $L=1000 \mu \mathrm{m}$. The optimum mesh size for which the pressure drop matches to within $0.3 \%$ of the value with the finest mesh size considered is highlighted. 


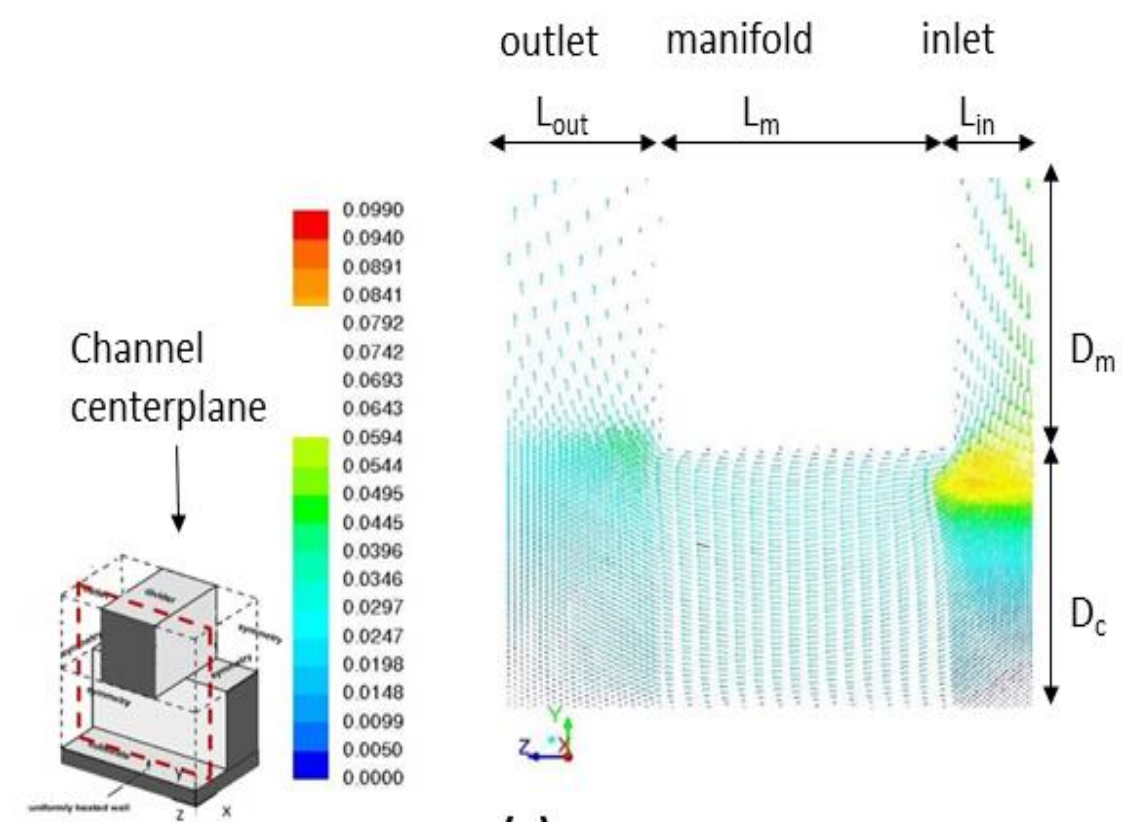

(a)

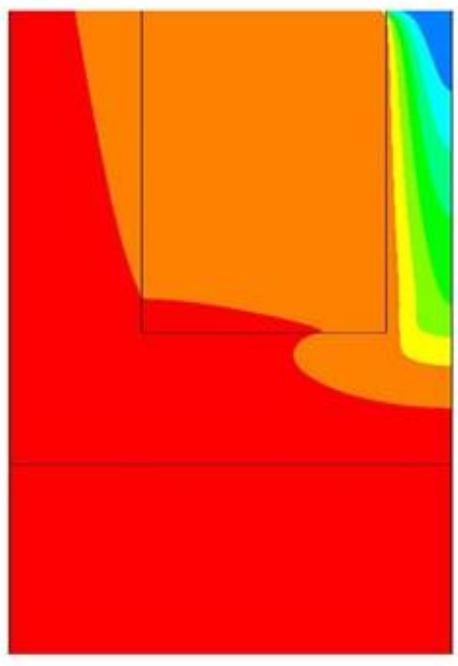

(b)

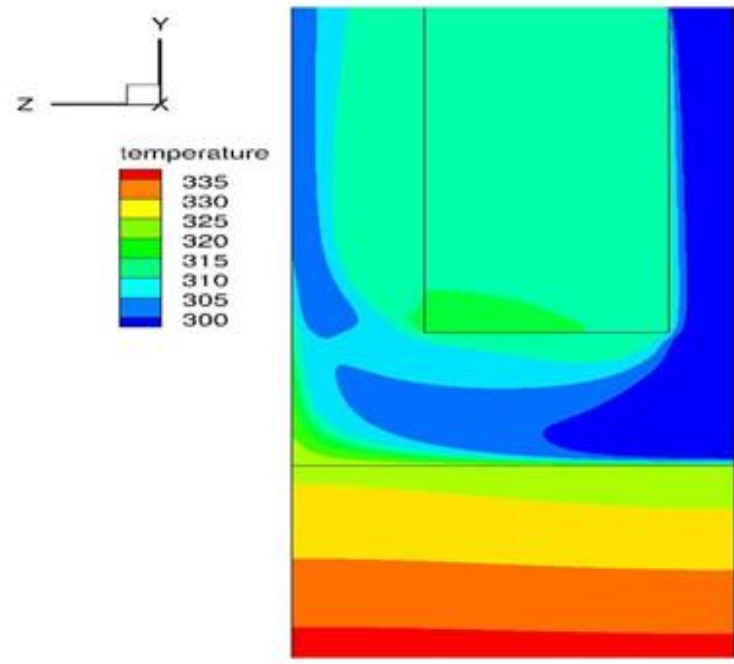

(c)

Figure 4: Velocity vectors and temperature contours for channel dimensions $\mathrm{W}_{\mathrm{c}}=40 \mu \mathrm{m}, \mathrm{D}_{\mathrm{c}}=200 \mu \mathrm{m}$, $\mathrm{D}_{\mathrm{m}}=500 \mu \mathrm{m}, \mathrm{L}_{\text {in }}=60 \mu \mathrm{m}, \mathrm{L}_{\text {out }}=120 \mu \mathrm{m}$ : (a) Velocity vectors at center plane of microchannel (plane shown in red dashed lines in the inset) for overall inlet mass flow rate $=0.5 \mathrm{~g} / \mathrm{s}$; (b) Temperature contours at center plane of microchannel for overall inlet mass flow rate of $0.5 \mathrm{~g} / \mathrm{s}$; and (c) Temperature contours at center plane of microchannel for overall inlet mass flow rate of $5.0 \mathrm{~g} / \mathrm{s}$. Velocity values are in $\mathrm{m} / \mathrm{s}$ and temperature values are in Kelvin. 


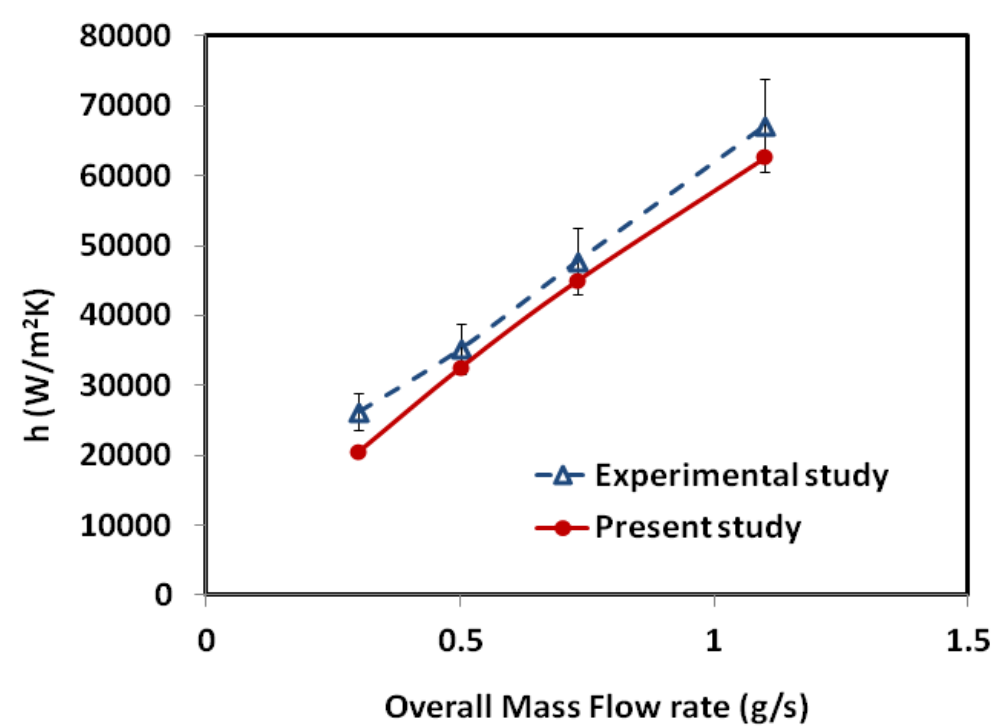

Figure 5: Heat transfer coefficient as a function of flow rate for a channel aspect ratio $=0.1$. Also shown are experimental results along with reported uncertainties from Kermani [9]. 


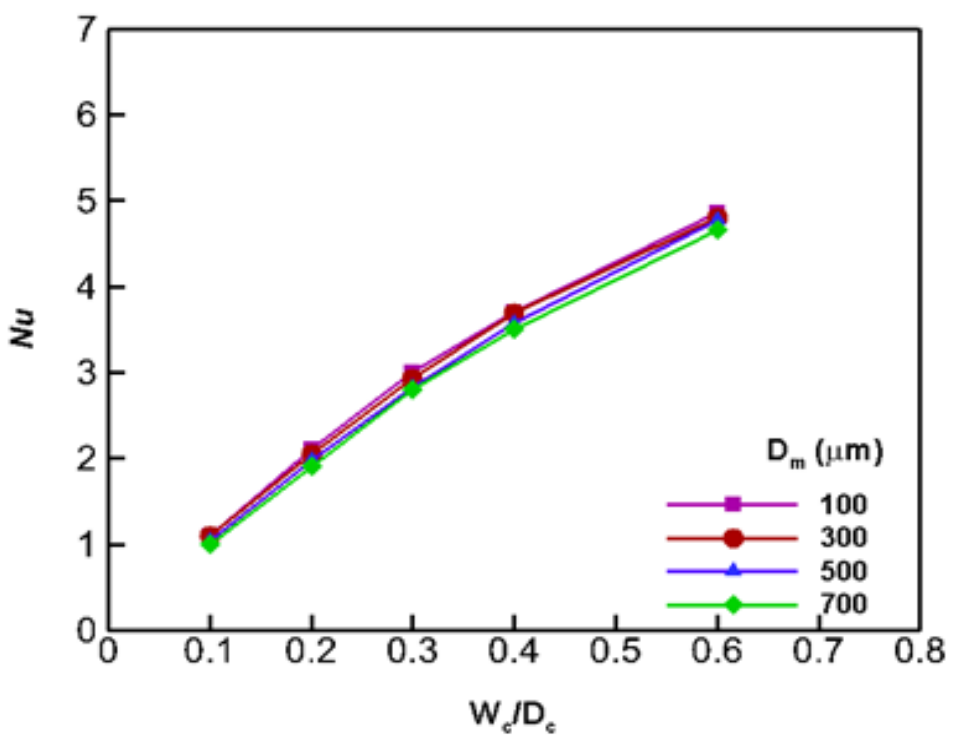

(a)

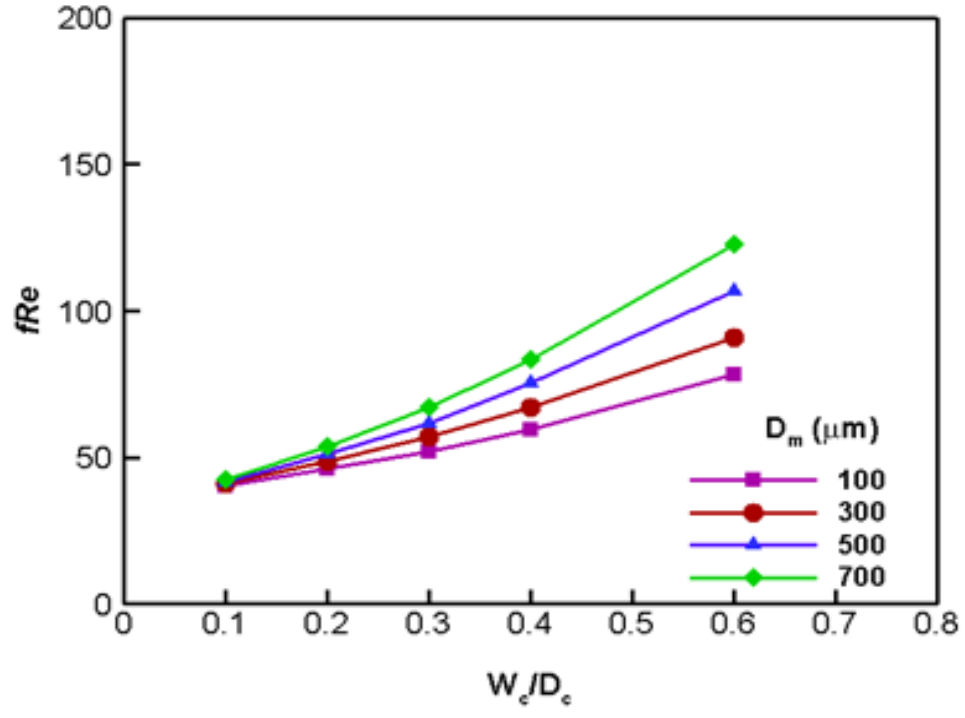

(b)

Figure 6: Effect of the geometric parameters on outputs: (a) $\mathrm{Nu}$, and (b) fRe, computed for the case of fixed coolant mass flow rate of $0.5 \mathrm{~g} / \mathrm{s}$. 

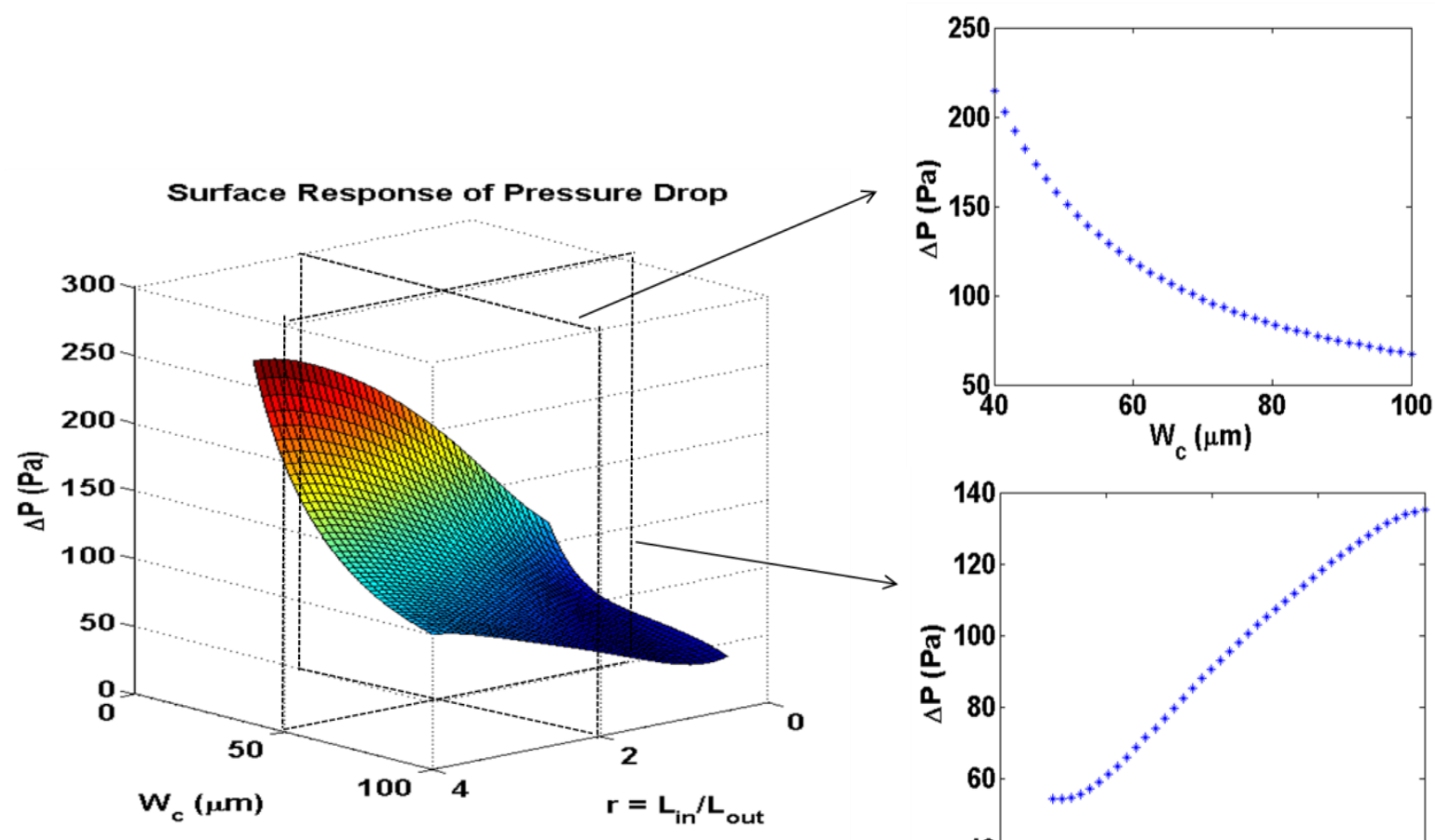

(a)
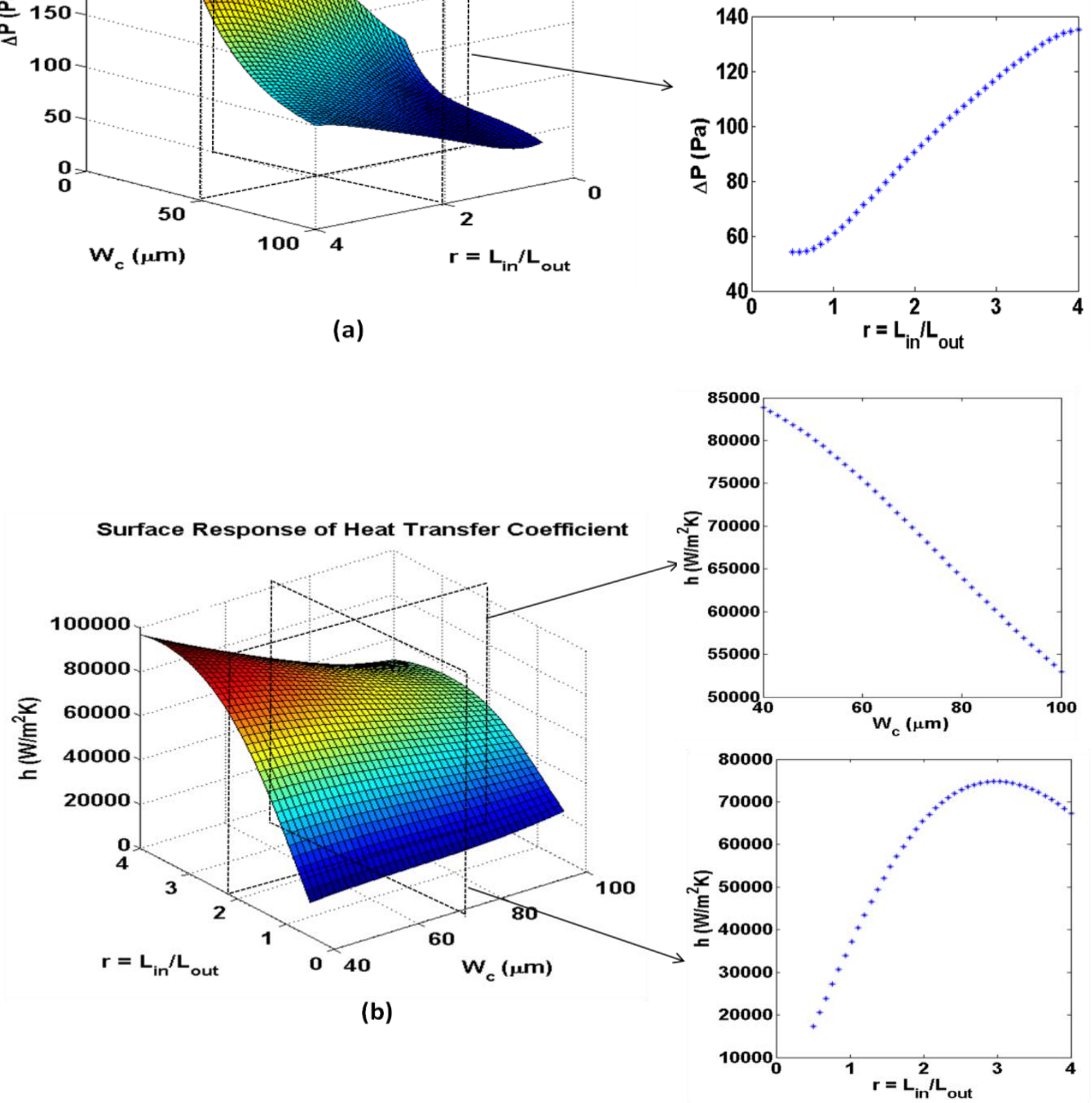

Figure 7: Representative response surfaces of (a) pressure drop, and (b) heat transfer coefficient, shown as a function of variation in channel width and manifold ratio. The insets show variation of the outputs relative to variation in each input parameter, obtained by holding the other input parameter at its mean value as indicated. 


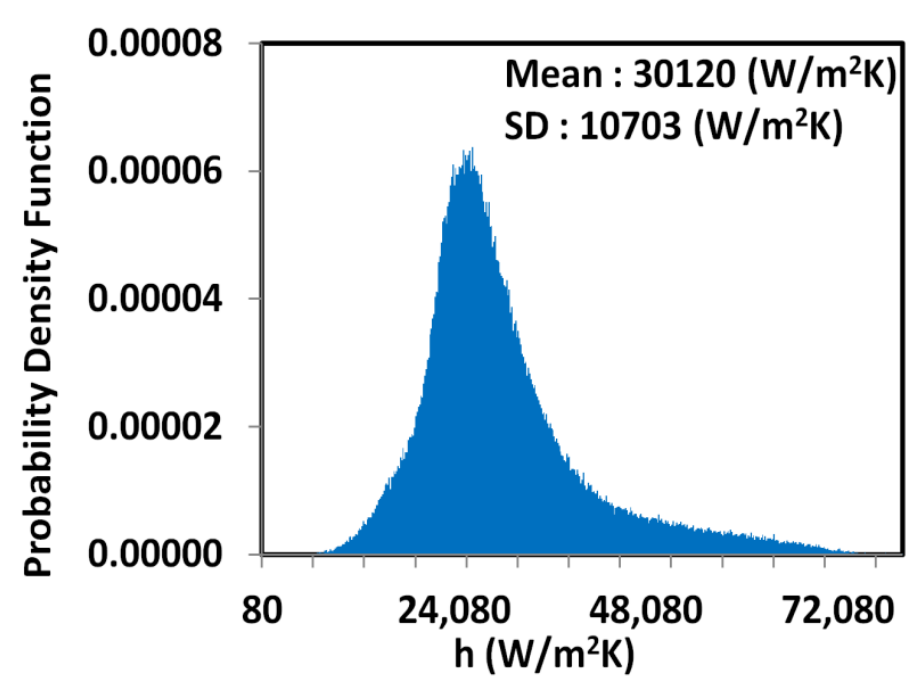

(a)

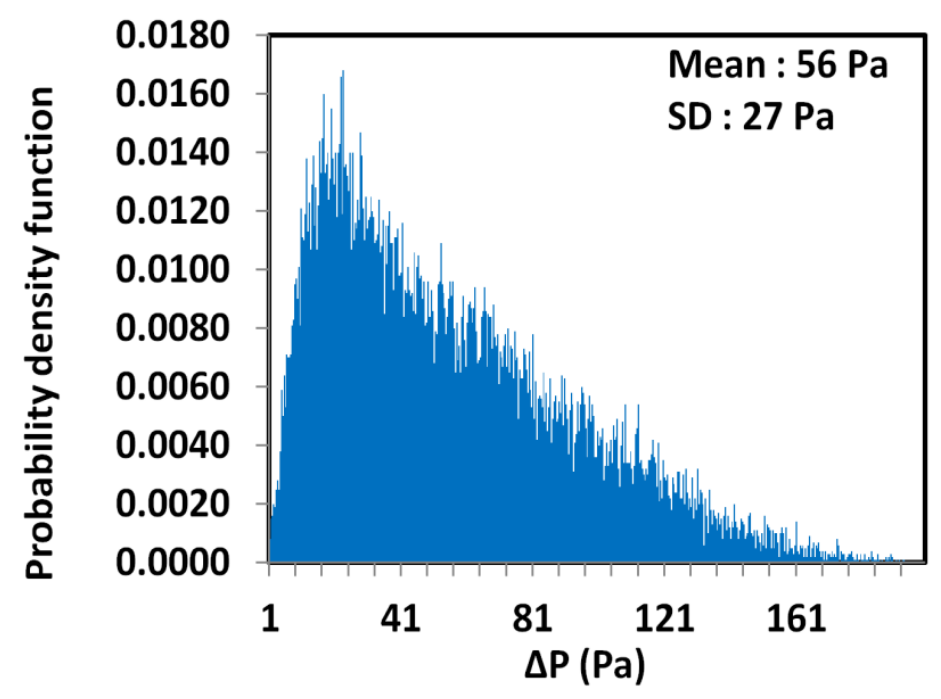

(b)

Figure 8: PDF of (a) heat transfer coefficient, and (b) pressure drop for uniformly distributed input parameters in Table 3. 


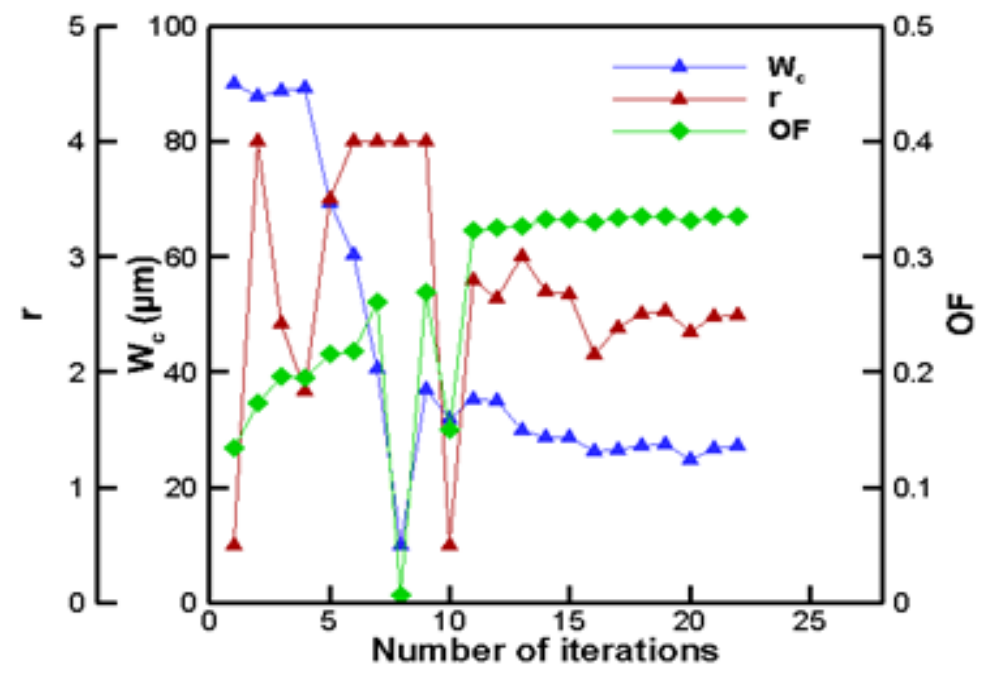

(a)

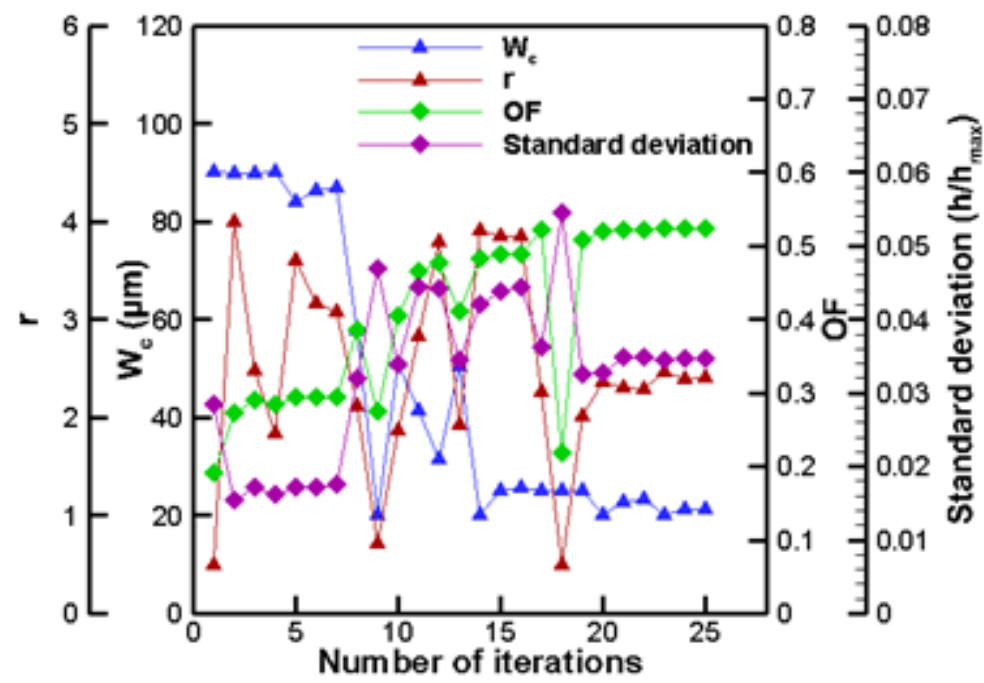

(b)

Figure 9: Convergence history of (a) deterministic optimization for $w_{1}=w_{2}=0.5$, and (b) probabilistic optimization for $w_{1}=0.7$ and $w_{2}=0.3$. 